Periodica Polytechnica Civil Engineering, 65(1), pp. 37-55, 2021

\title{
Optimal Design of Passive and Active Control Systems in Seismic-excited Structures Using a New Modified TLBO
}

\author{
Saeed Hosseinaei ${ }^{1}$, Mohammad Reza Ghasemi ${ }^{1}$, Sadegh Etedali ${ }^{* 2}$ \\ ${ }^{1}$ Department of Civil Engineering, University of Sistan and Baluchestan, P.O. Box 98155-987, Zahedan, Iran \\ ${ }^{2}$ Department of Civil Engineering, Birjand University of Technology, P.O. Box 97175-569, Birjand, Iran \\ * Corresponding author, e-mail: etedali@birjandut.ac.ir
}

Received: 21 May 2020, Accepted: 30 July 2020, Published online: 01 October 2020

\begin{abstract}
Vibration control devices have recently been used in structures subjected to wind and earthquake excitations. The optimal design problems of the passive control device and the feedback gain matrix of the controller for the seismic-excited structures are some attractive problems for researches to develop optimization algorithms with the advancement in terms of simplicity, accuracy, speed, and efficacy. In this paper, a new modified teaching-learning-based optimization (TLBO) algorithm, known as MTLBO, is proposed for the problems. For some benchmark optimization functions and constrained engineering problems, the validity, efficacy, and reliability of the MTLBO are firstly assessed and compared to other optimization algorithms in the literature. The undertaken statistical indicate that the MTLBO performs better and reliable than some other algorithms studied here. The performance of the MTLBO will then be explored for two passive and active structural control problems. It is concluded that the MTLBO algorithm is capable of giving better results than conventional TLBO. Hence, its utilization as a simple, fast, and powerful optimization tool to solve particular engineering optimization problems is recommended.
\end{abstract}

Keywords

optimization, TLBO, modified TLBO, engineering optimization, structural control optimization

\section{Introduction}

Vibration control devices have been successfully used for vibration mitigation of buildings and bridges against dynamic loads such as strong winds and, earthquakes. The optimal tuning of the parameters of the passive control device, supplemented to the structures, has a direct effect on the seismic responses of the structures. Some researchers attempt to utilize or develop meta-heuristic optimization algorithms in this regard. Etedali et al. [1] utilized a cuckoo search (CS) optimization algorithm for the optimal design of friction tuned mass damper (FTMD). Fahimi Farzam and Kaveh [2] utilized colliding bodies optimization (CBO) for optimum design of TMD in the frequency domain. Ghasemi et al. [3] used an improved ideal gas molecules movements (IGMM) in SMA dampers for vibration control of Jacket-type offshore structures. The optimal design of rotational friction dampers using particle swarm optimization (PSO) is studied in [4]. Kaveh et al. [5] compared the $\mathrm{H}_{2}$ and $\mathrm{H}_{\infty}$ norm of roof displacement transfer function as the objective functions for optimum design of TMD under near-fault and far-fault earthquake motions.
A robust optimum design of tuned mass damper inerter (TMDI) is also proposed by Kaveh et al. [6]. The design of controllers has a key role in the successful implementation of the smart structures to tune the control force of the actuator. Some optimization algorithms such as GA [7], IGMM [8], CSS [9], and gases Brownian motion optimization [10] have recently given attention to the optimal design of controllers in the seismic-excited structures.

There are different optimization algorithms inspired by the swarm intelligence and evolutionary computations in the literature. Some of these algorithms include GA, PSO, search and rescue (SAR) and ideal gas molecular movement (IGMM). The GA has been inspired by Darwin's evolution theory focusing on the survival of the fittest [11]. PSO imitates the behavior of a bird flock or fish to search for food [12]. SAR imitates the explorations which were carried out by humans during search and rescue operations [13] and IGMM is inspired by the movements of gas molecules [14]. Recently, some new optimization algorithms such as Echolocation Search Algorithm [15], 
enhanced artificial coronary circulation system [16], natural forest regeneration [17], hybrid invasive weed optimization-shuffled frog-leaping [18], quantum evolutionary algorithm [19] and search and rescue optimization algorithm [20], have been also proposed for civil engineering optimization problems.

Recently, Rao et al. [21] have developed a new optimization algorithm called Teaching-Learning-based optimization (TLBO) in which the focus is on the concept of the scenario of classroom teaching. The TLBO works based on the effect of a teacher on the performance of learners in the classroom. This performance can be measured by the grades achieved by the learner. In this philosophy, the teacher, as a knowledge supplier, is the person who can lead the student to obtain better results. A better teacher makes learners achieve better results. The superiority of the TLBO algorithm to other optimization algorithms is reported in [22]. The advantages of TLBO in terms of better understanding, easy implementation, and the need for a small number of parameters to operate have made it one of the most commonly used optimization algorithms. Recently, Nayak et al. [23] proposed an effective approach integration of the Taguchi method (TM), Adaptive neuro-fuzzy inference system (ANFIS) and TLBO for CNC turning optimization of S45C carbon steel. Dang et al. [24] also utilized a TLBO algorithm for solving a multi-objective optimization design for a new linear compliant mechanism.

In the present paper, a new modification on the basic TLBO, known as MTLBO, is proposed. For this purpose, an extra term is added to basic TLBO in the both teacher phase and learner phase to speed up the convergence rate, a descriptive detail of which is given later in this study. The performance of the proposed MTLBO algorithm is investigated in comparison with $\mathrm{PSO}, \mathrm{DE}$, and $\mathrm{ABC}$ for different benchmark optimization functions followed by its application on some engineering benchmark optimization problems. To the best knowledge of the authors, no up-to-date study is found to utilize the TLBO in structural control problems. Hence, this paper also applies the new modification of the basic TLBO for two structural control problems. For this purpose, the optimal design problems of TMD device as a passive control device and optimal tuning of the feedback gain matrix of the controller in an active tendon system for a seismic-excited structure are addressed in this study.

The remainder of the paper is organized as follows: Section 2 gives a brief description of TLBO. The MTLBO algorithm is proposed in Section 3. Section 4 is divided into three subsections. Considering the benchmark optimization functions, the performance of the proposed MTLBO algorithm is compared with some other optimization algorithms in the first subsection. In the second subsection, examples of engineering benchmark problems are solved using MTLBO and its performance is compared to TLBO and other optimization techniques. The proposed MTLBO algorithm is applied to two structural control problems in the third subsection. Finally, the conclusion of the present paper is reported in Section 5.

\section{Teaching-Learning-Based Optimization (TLBO)}

In a population-based method such as TLBO, a series of solutions have been used for progress to get the global solution. TLBO is based on the effect of a teacher on the performance of learners in the class. TLBO algorithm consists of two main phases including the teacher phase and learner phase. The teacher phase refers to the occurrence of the learning process due to teacher role while the learner Phase deals with the happening of learning as a result of interactions between learners. Rao has explained the basic steps of TLBO. The teacher phase refers to the occurrence of the learning process due to teacher role while the learner Phase deals with the happening of learning as a result of interactions between learners. The basic phases of the TLBO are as follows [22].

\subsection{Teacher phase}

As can be seen from Fig. 1, a good teacher can improve the mean value of the scores obtained by the learners from $M_{A}$ to $M_{B}$. A good teacher is a person who promotes the knowledge of learners. In practice, it is evident that the

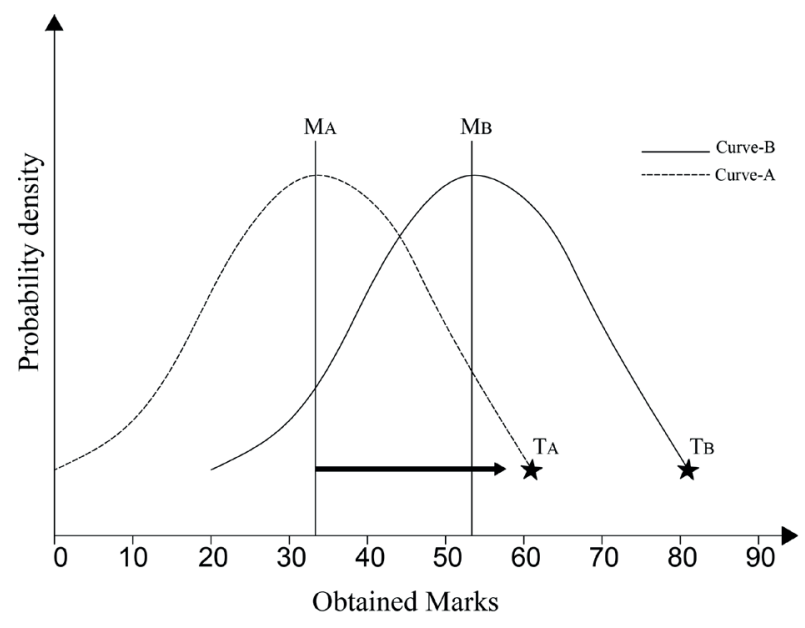

Fig. 1 Model for the distribution of marks obtained for a group of learners [22] 
teacher can improve the mean score of the class partially so that the extent depends on the overall ability of the class members and so factors are involved.

It is assumed that the mean value of the score and the teacher at the $i^{\text {th }}$ iteration are denoted by $M_{i}$ and $T_{i}$, respectively. In the teacher phase, $T_{i}$ will try to enhance the value of the mean $M_{i}$ to its own level, so that the new mean is denoted by $M_{n e w}$. Depending on the difference between existing mean and $M_{n e w}$, the solution can be updated using the following equation:

$$
\text { Difference_Mean }_{i}=r_{i}\left(M_{\text {new }}-T_{F} M_{i}\right) \text {, }
$$

where $T_{F}$ refers to the teaching factor which attempts to change the mean value. Also, $r_{i}$ refers to a random number in the interval $[0,1]$. The value of $T_{F}$ is set as either 1 or 2 . The following equation is used to modify the existing solution:

$$
X_{\text {new }, i}=X_{\text {old }, i}+\text { Difference_Mean }{ }_{i}
$$

\subsection{Learner phase}

The promotion of learners in the learning process is done in two various ways: Learning from the teacher and the interactions among learners. The interaction among learners occurs through discussions, presentations, formal communications, etc. In other words, a learner can learn new things when a knowledgeable learner gives more information about a certain subject. The modification of learner can be expressed as Algorithm 1.

\section{Modified Teaching-Learning-Based Optimization (MTLBO)}

In this Section, a new modified TLBO algorithm is introduced. For this aim, two extra terms in the both teacher and learner phases of the conventional TLBO algorithm are added. An optimization algorithm includes exploration and exploitation phases. In the exploration phase, the

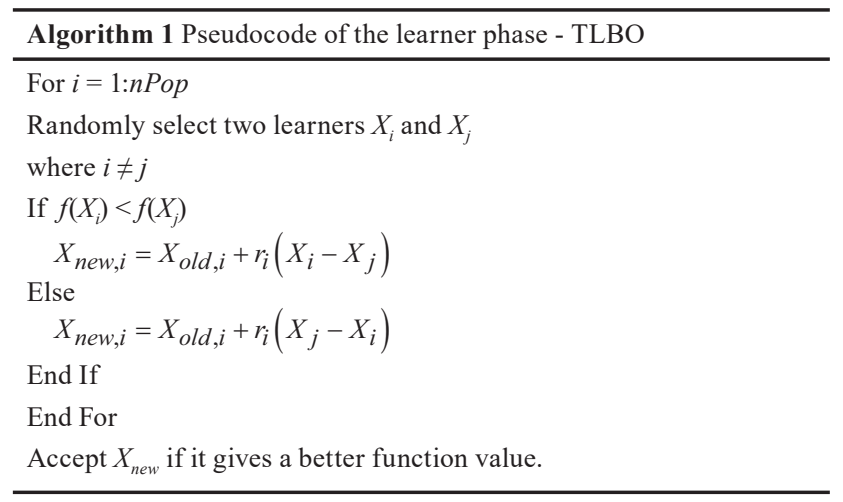

Where $n P o p$ is the number of population. entire answer space is searched and it is finally found the region that includes the best solutions. In the exploitation phase, the region that was found in the exploration phase is searched. In fact, in the exploitation phase, the searching operation is done more precisely in the smaller region. The conventional TLBO and the proposed MTLBO have both phases in the teacher and learner phases, respectively. However, a new term is added to the teacher phase which results in more space is sought for finding a better solution than the conventional TLBO. Moreover, in the learner phase, for a more detailed search and increase the speed of finding the best solution, changes or mobility in the search space are decreased to half of the previous values that were happened in the conventional TLBO. It makes better exploitation in the search spaces and gets more diversity. The modification of the conventional TLBO algorithm is proposed as follows:

\subsection{Teacher phase}

As previously mentioned, the conventional TLBO algorithm in the teacher phase aims to bring the mean score closer to the teacher score. Therefore, in this phase, mobility is toward the best learner (teacher). In addition to the movement towards the best learner (teacher), established in the conventional TLBO algorithm, to increase the speed of students' learning, it is also proposed in the MTLBO algorithm that they get away from the worst learner for more space is sought for finding a better solution. For this purpose, in the MTLBO algorithm, at first, the students are arranged in the worst to the best (teacher) order. Based on the mentioned modification, an extra term can be added to the teacher phase of the TLBO. From a mathematical point of view, the modified teacher phase of the basic TLBO can be expressed as the following equation:

$$
\begin{aligned}
& X_{\text {new }, i}=X_{\text {old }, i}+\text { rand } *\left(X_{\text {Teacher }}-T_{F} * \text { Mean }\right) \\
& +\operatorname{rand}^{*}\left(\text { Mean }-X_{\text {Worst }}\right),
\end{aligned}
$$

where $X_{\text {Worst }}$ is the worst grade among all the students. Accept $X_{n e w}$, if it gives a better function value.

\subsection{Learner phase}

The conceptual analysis of the TLBO algorithm makes clear that as the learner learns more, the solution becomes better. The learning performance of the students can be enhanced via the reduction of changes or mobility in the search space to half of the previous values in the conventional TLBO. In other words, it is proposed that only half 
of the current solutions (dimensions) are changed in the MTLBO. It makes better exploitation in the search spaces and gets more diversity. The modified learner phase can be stated as Algorithm 2.

\section{Numerical studies}

The efficacy of the proposed MTLBO algorithm is compared with other evolutionary optimization algorithms including GA, PSO, ABC, and DE algorithms for different basic benchmark optimization functions. Then, the performance of the MTLBO algorithm is compared with the basic TLBO algorithm for CEC-2005 benchmark optimization functions. In the end, the MTLBO is developed for the optimal design of TMD parameters and optimal tuning of the feedback gain matrix of the controller in an active tendon system.

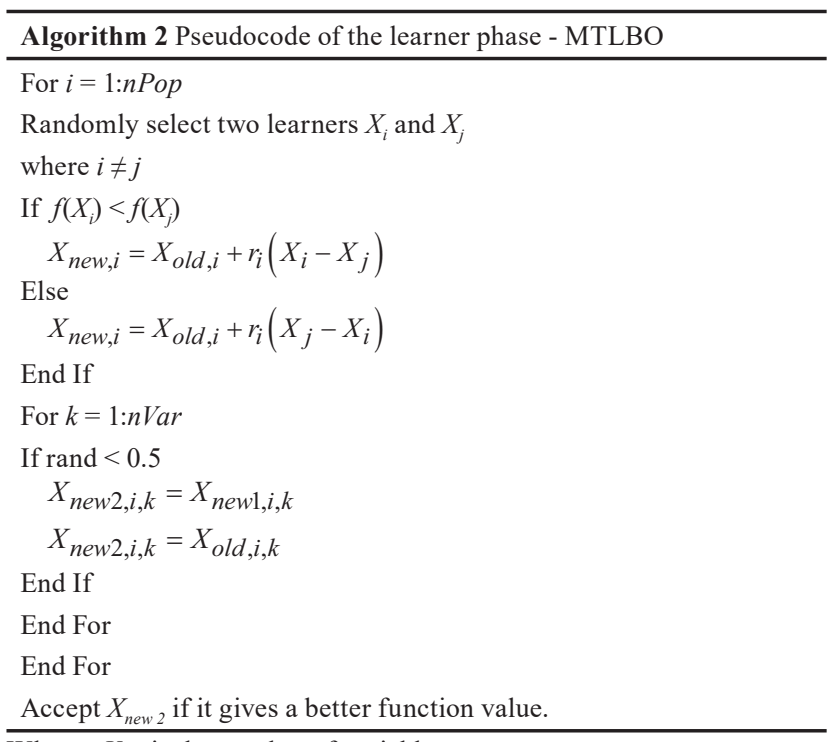

\subsection{Benchmark optimization function}

Six benchmark optimization functions as multimodal problems are chosen to test the ability of the global search of different optimization algorithms. The benchmark optimization functions are summarized in Table 1.

\subsubsection{Experiments $A$}

The efficacy of the MTLBO algorithm in comparison with OEA, HPSO-TVAC, CLPSO, APSO, OLPSO-L, OLPSO-G for the benchmark optimization functions, defined in Table 1, are summarized in Table 2. For this purpose, the mean and the standard deviation (SD) criteria are inserted in this table. The results of OEA, HPSOTVAC, CLPSO, and APSO, OLPSO-L, OLPSO-G are reported in [25]. For a fair comparison, the numbers of population (NPop) for TLBO and MTLBO are considered as 5 and the maximum iteration is considered as 5000. 10 runs are assigned for these computations. The number of function evaluations (NFE) for each function is mentioned in the table. Also, the final performance of the MTLBO respect to other algorithms is reported in the last three rows of the Table in terms of worse, better and similar performance. "-", "+", and " $\approx$ " denote that the performance of the corresponding algorithm is worse than, better than, and similar to that of MTLBO, respectively. NA is used for Not Available. The results show that the MTLBO performs better than OEA, HPSO-TVAC, CLPSO, APSO, OLPSO-L and OLPSO-G for all test functions. For most problems, the results are given with less NFE than other algorithms. Similar results are given by the original TLBO and the MTLBO for the most test functions.

Table 1 Benchmark test functions

\begin{tabular}{|c|c|c|c|}
\hline Test function & Formulation & Search range & Minimum value \\
\hline Sphere & $f_{1}(x)=\sum_{i=1}^{D} x_{i}^{2}$ & {$[-100,100]^{\mathrm{D}}$} & 0 \\
\hline Schwefel 2.22 & $f_{2}(x)=\sum$ & {$[-10,10]^{\mathrm{D}}$} & 0 \\
\hline Step & $f_{3}(x)=\sum_{i=1}^{D}\left[\left|x_{i}+0.5\right|\right]^{2}$ & {$[-100,100]^{\mathrm{D}}$} & 0 \\
\hline Schwefel 1.2 & $f_{4}(x)=\sum_{i=}^{D}$ & {$[-100,100]^{\mathrm{D}}$} & 0 \\
\hline Ackley & $f_{5}(x)=-20 \exp \left(-0.2 * \sqrt{\frac{1}{D}} \sum_{i=1}^{D} x_{i}^{2}\right)-\exp \left(\frac{1}{D} \sum_{i=1}^{D} \cos \left(2 \pi x_{i}\right)\right)+20+e$ & {$[-32,32]^{\mathrm{D}}$} & 0 \\
\hline Griewank & $f_{6}(x)=\frac{1}{4000} \sum_{i=1}^{D} x_{i}^{2}-\prod_{i}^{D} \cos \left(\frac{x_{i}}{\sqrt{i}}\right)+1$ & {$[-600,600]^{\mathrm{D}}$} & 0 \\
\hline
\end{tabular}




\subsubsection{Experiments B}

The experiments of this group compare the performance of the MTLBO algorithm with those given by SaDE, jDE, JADE, CoDE, EPSDE for the benchmark functions described in Table 1. The results of these algorithms are directly taken from [25]. The results are inserted in Table 3. A similar result is obtained for this experiment. The superiority of the MTLBO than other algorithms are observed.

\subsubsection{Experiments $C$}

The experiments of this group validate the performance of the MTLBO algorithm in comparison with CABC, GABC, RABC and IABC for solving the mentioned six benchmark optimization functions. The results of these algorithms are given by [25]. The corresponding results for each test function are shown in Table 4. Similar to the results in experiments $\mathrm{A}$ and $\mathrm{B}$, it is found that the MTLBO performs better than other optimization algorithms to find the best solution for the mentioned benchmark test functions.

\subsubsection{CEC-2005 benchmark optimization functions}

In Tables 2-4, the optimal result of each function is zero and it is concluded that both basic TLBO and the MTLBO give better performance than other algorithms in terms of mean and SD.
Also, it is found that the basic TLBO and MTLBO result in the same results in most benchmark optimization functions. Considering different CEC-2005 benchmark optimization functions that have the non-zero optimal result, a comparison between the performance of the MTLBO and TLBO is interesting. Table 5 shows the CEC-2005 benchmark optimization functions. For a fair comparison, the number of runs is considered as 25 and the number of function evaluations is $10000^{*} \mathrm{D}$ where $\mathrm{D}$ is dimensionalities of the problems. Also, the population sizes for both TLBO and MTLBO are considered as 10 . The average error for 25 functions is indicated in Table 6. Also, the convergence rate diagrams for functions $1,6,11,16$, and 21 are illustrated in Fig. 2. In Table 6, it can be seen that the MTLBO has a less mean of error than conventional TLBO in all functions except functions 3 and 20. Furthermore, Fig. 2 shows that the MTLBO converges more quickly than the TLBO to the optimal solutions. Consequently, the MTLBO gives better performance and reliable results than the TLBO.

\subsection{Engineering optimization problems}

The performance of the MTLBO algorithm is also verified for some engineering optimization problems. Four benchmark engineering problems are selected for this purpose and the penalty function method approach is utilized to handle the defined constraints for the problems as the following pseudo-cost function:

Table 2 Performance of MTLBO, OEA, HPSO-TVAC, CLPSO, APSO, OLPSO-L and OLPSO-G

\begin{tabular}{|c|c|c|c|c|c|c|c|c|c|}
\hline Function & & OEA & HPSO-TVAC & CLPSO & APSO & OLPSO-L & OLPSO-G & TLBO & MTLBO \\
\hline Sphere & $\begin{array}{l}\text { Mean } \\
\text { SD } \\
\text { NFE }\end{array}$ & $\begin{array}{c}2.48 \mathrm{e}-30(-) \\
1.128 \mathrm{e}-29 \\
1.5^{*} 10^{5}\end{array}$ & $\begin{array}{c}3.38 \mathrm{e}-41(-) \\
8.50 \mathrm{e}-41 \\
1.5^{*} 10^{5}\end{array}$ & $\begin{array}{c}1.89 \mathrm{e}-19(-) \\
1.49 \mathrm{e}-19 \\
1.5^{*} 10^{5}\end{array}$ & $\begin{array}{c}1.45 \mathrm{e}-150(-) \\
5.73 \mathrm{e}-150 \\
1.5^{*} 10^{5}\end{array}$ & $\begin{array}{c}1.11 \mathrm{e}-38(-) \\
1.28 \mathrm{e}-28 \\
1.5 * 10^{5}\end{array}$ & $\begin{array}{c}4.12 \mathrm{e}-54(-) \\
6.34 \mathrm{e}-54 \\
1.5^{*} 10^{5}\end{array}$ & $\begin{array}{c}4.9 e-321 \\
0 \\
5 * 10^{4}\end{array}$ & $\begin{array}{c}4.9 e-321 \\
0 \\
5 * 10^{4}\end{array}$ \\
\hline $\begin{array}{l}\text { Schwefel } \\
2.22\end{array}$ & $\begin{array}{l}\text { Mean } \\
\text { SD } \\
\text { NFE }\end{array}$ & $\begin{array}{c}2.07 \mathrm{e}-13(-) \\
2.44 \mathrm{e}-12 \\
2.0 * 10^{5}\end{array}$ & $\begin{array}{c}6.92 \mathrm{e}-23(-) \\
6.89 \mathrm{e}-23 \\
2.0 * 10^{5}\end{array}$ & $\begin{array}{c}1.01 \mathrm{e}-13(-) \\
6.54 \mathrm{e}-14 \\
2.0^{*} 10^{5}\end{array}$ & $\begin{array}{c}5.15 \mathrm{e}-84(-) \\
1.44 \mathrm{e}-83 \\
2.0^{*} 10^{5}\end{array}$ & $\begin{array}{c}7.67 \mathrm{e}-22(-) \\
5.63 \mathrm{e}-22 \\
2.0 * 10^{5}\end{array}$ & $\begin{array}{c}9.85 \mathrm{e}-30(-) \\
1.01 \mathrm{e}-29 \\
2.0 * 10^{5}\end{array}$ & $\begin{array}{c}4.9 e-321 \\
0 \\
5 * 10^{4}\end{array}$ & $\begin{array}{c}4.9 e-321 \\
0 \\
5 * 10^{4}\end{array}$ \\
\hline Step & $\begin{array}{l}\text { Mean } \\
\text { SD } \\
\text { NFE }\end{array}$ & $\begin{array}{c}0(\sim) \\
0 \\
1.0^{*} 10^{5}\end{array}$ & $\begin{array}{c}0(\sim) \\
0 \\
1.0 * 10^{5}\end{array}$ & $\begin{array}{c}0(\sim) \\
0 \\
1.0 * 10^{5}\end{array}$ & $\begin{array}{c}0(\sim) \\
0 \\
1.0 * 10^{5}\end{array}$ & $\begin{array}{l}\text { NA } \\
\text { NA } \\
\text { NA }\end{array}$ & $\begin{array}{l}\text { NA } \\
\text { NA } \\
\text { NA }\end{array}$ & $\begin{array}{c}0 \\
0 \\
5 * 10^{4}\end{array}$ & $\begin{array}{c}0 \\
0 \\
5 * 10^{4}\end{array}$ \\
\hline $\begin{array}{l}\text { Schwefel } \\
1.2\end{array}$ & $\begin{array}{l}\text { Mean } \\
\text { SD } \\
\text { NFE }\end{array}$ & $\begin{array}{c}5.43 \mathrm{e}-17(-) \\
1.68 \mathrm{e}-16 \\
1.0 * 10^{5}\end{array}$ & $\begin{array}{c}2.39(-) \\
3.71 \\
1.0 * 10^{5}\end{array}$ & $\begin{array}{c}2.57 \mathrm{e}-1(-) \\
6.64 \mathrm{e}-11 \\
1.0 * 10^{5}\end{array}$ & $\begin{array}{c}5.8 \mathrm{e}-15(-) \\
1.01 \mathrm{e}-14 \\
1.0 * 10^{5}\end{array}$ & $\begin{array}{c}0(\sim) \\
0 \\
1.0 * 10^{5}\end{array}$ & $\begin{array}{c}1.07(-) \\
0.99 \\
1.0 * 10^{5}\end{array}$ & $\begin{array}{c}1 \mathrm{e}-323 \\
4.4238 \\
5 * 10^{4}\end{array}$ & $\begin{array}{c}9.2076 \mathrm{e}-281 \\
0 \\
5 * 10^{4}\end{array}$ \\
\hline Ackley & $\begin{array}{l}\text { Mean } \\
\text { SD } \\
\text { NFE }\end{array}$ & $\begin{array}{c}5.34 \mathrm{e}-14(-) \\
2.94 \mathrm{e}-13 \\
5.0 * 10^{4}\end{array}$ & $\begin{array}{c}2.06 \mathrm{e}-10(-) \\
9.45 \mathrm{e}-10 \\
5.0^{*} 10^{4}\end{array}$ & $\begin{array}{c}2.01 \mathrm{e}-12(-) \\
9.22 \mathrm{e}-13 \\
5.0^{*} 10^{4}\end{array}$ & $\begin{array}{c}1.11 \mathrm{e}-14(-) \\
3.55 \mathrm{e}-15 \\
5.0 * 10^{4}\end{array}$ & $\begin{array}{c}4.14 \mathrm{e}-5(-) \\
0 \\
5.0 * 10^{4}\end{array}$ & $\begin{array}{c}7.98 \mathrm{e}-15(-) \\
2.03 \mathrm{e}-15 \\
5.0 * 10^{4}\end{array}$ & $\begin{array}{c}4.2633 \mathrm{e}-15 \\
1.498 \mathrm{e}-15 \\
5 * 10^{4}\end{array}$ & $\begin{array}{c}3.5527 \mathrm{e}-15 \\
0 \\
5 * 10^{4}\end{array}$ \\
\hline Griewank & $\begin{array}{l}\text { Mean } \\
\text { SD } \\
\text { NFE }\end{array}$ & $\begin{array}{c}1.32 \mathrm{e}-02(-) \\
1.56 \mathrm{e}-02 \\
5 * 10^{4}\end{array}$ & $\begin{array}{c}1.07 \mathrm{e}-02(-) \\
1.14 \mathrm{e}-02 \\
5 * 10^{4}\end{array}$ & $\begin{array}{c}6.45 \mathrm{e}-13(-) \\
2.07 \mathrm{e}-12 \\
5 * 10^{4}\end{array}$ & $\begin{array}{c}1.67 \mathrm{e}-02(-) \\
2.41 \mathrm{e}-02 \\
5 * 10^{4}\end{array}$ & $\begin{array}{c}0(\sim) \\
0 \\
5 * 10^{4}\end{array}$ & $\begin{array}{c}4.83 e-03(-) \\
8.63 e-03 \\
5 * 10^{4}\end{array}$ & $\begin{array}{c}0 \\
0 \\
5 * 10^{4}\end{array}$ & $\begin{array}{c}0 \\
0 \\
5 * 10^{4}\end{array}$ \\
\hline+ & 5 & 5 & 5 & 5 & 3 & 5 & 1 & & \\
\hline- & 0 & 0 & 0 & 0 & 0 & 0 & 1 & & \\
\hline$\approx$ & 1 & 1 & 1 & 1 & 2 & 0 & 4 & & \\
\hline
\end{tabular}


Table 3 Performance of MTLBO, JADE, jDE, SaDE, CoDE, and EPSDE

\begin{tabular}{|c|c|c|c|c|c|c|c|c|}
\hline Function & & $\mathrm{SaDE}$ & $\mathrm{jDE}$ & JADE & CoDE & EPSDE & TLBO & MTLBO \\
\hline \multirow{3}{*}{ Sphere } & Mean & $4.5 \mathrm{e} 20(-)$ & $2.5 \mathrm{e}-28(-)$ & $1.8 \mathrm{e}-60(-)$ & $1.12 \mathrm{e}-31(-)$ & $1.53 \mathrm{e}-85(-)$ & $4.9 \mathrm{e}-321$ & $4.9 \mathrm{e}-321$ \\
\hline & SD & $1.9 \mathrm{e}-14$ & $3.5 \mathrm{e}-28$ & $8.4 \mathrm{e}-60$ & $3.45-31$ & $9.01 \mathrm{e}-86$ & 0 & 0 \\
\hline & NFE & $1.5^{*} 10^{5}$ & $1.5^{*} 10^{5}$ & $1.5^{*} 10^{5}$ & $1.5^{*} 10^{5}$ & $1.5^{*} 10^{5}$ & $5 * 10^{4}$ & $5 * 10^{4}$ \\
\hline \multirow{3}{*}{$\begin{array}{l}\text { Schwefel } \\
2.22\end{array}$} & Mean & $1.9 \mathrm{e} 14(-)$ & $1.5 \mathrm{e}-23(-)$ & $1.8 \mathrm{e}-25(-)$ & $1.22 \mathrm{e}-23(-)$ & $3.18 \mathrm{e}-54(-)$ & $4.9 \mathrm{e}-321$ & $4.9 \mathrm{e}-321$ \\
\hline & SD & $1.1 \mathrm{e}-14$ & $1.0 \mathrm{e}-23$ & $8.8 \mathrm{e}-25$ & $3.90 e-23$ & $3.11 \mathrm{e}-54$ & 0 & 0 \\
\hline & NFE & $2.0^{*} 10^{5}$ & $2.0 * 10^{5}$ & $2.0^{*} 10^{5}$ & $2.0^{*} 10^{5}$ & $2.0^{*} 10^{5}$ & $5 * 10^{4}$ & $5 * 10^{4}$ \\
\hline \multirow{3}{*}{ Step } & Mean & $9.3 \mathrm{e}+02(-)$ & $1.0 \mathrm{e}+03(-)$ & $2.9 \mathrm{e}+0(-)$ & $3.00 \mathrm{e}+00(-)$ & $0(\sim)$ & 0 & 0 \\
\hline & SD & $1.8 \mathrm{e}+02$ & $2.2 \mathrm{e}+02$ & $1.2 \mathrm{e}+0$ & $1.90 \mathrm{E}+00$ & 0 & 0 & 0 \\
\hline & NFE & $1.0 * 10^{4}$ & $1.0 * 10^{4}$ & $1.0^{*} 10^{4}$ & $1.0^{*} 10^{4}$ & $1.0 * 10^{4}$ & $5 * 10^{4}$ & $5 * 10^{4}$ \\
\hline \multirow{3}{*}{$\begin{array}{l}\text { Schwefel } \\
1.2\end{array}$} & Mean & $1.2 \mathrm{e}-03(-)$ & $1.5 \mathrm{e}-04(-)$ & $1.0 \mathrm{e}-04(-)$ & $1.21 \mathrm{e}-01(-)$ & $0(\sim)$ & $1 e-323$ & $9.2076 \mathrm{e}-281$ \\
\hline & $\mathrm{SD}$ & $6.5 \mathrm{e}-04$ & $2.0 \mathrm{e}-04$ & $6.0 \mathrm{e}-05$ & $3.89 \mathrm{e}-02$ & 0 & 4.4238 & 0 \\
\hline & NFE & $1.0^{*} 10^{5}$ & $1.0 * 10^{5}$ & $1.0 * 10^{5}$ & $1.0 * 10^{5}$ & $1.0^{*} 10^{5}$ & $5 * 10^{4}$ & $5^{*} 10^{4}$ \\
\hline \multirow{3}{*}{ Ackley } & Mean & $2.7 \mathrm{e}-03(-)$ & $3.5 \mathrm{e}-04(-)$ & $8.2 \mathrm{e}-10(-)$ & $1.18 \mathrm{e}-04(-)$ & $1.94 \mathrm{e}-2(-)$ & $4.2633 \mathrm{e}-15$ & $3.5527 \mathrm{e}-15$ \\
\hline & SD & $5.1 \mathrm{e}-04$ & $1.0 \mathrm{e}-04$ & $6.9 \mathrm{e}-10$ & $4.90 \mathrm{e}-04$ & $8.90 \mathrm{e}-4$ & $1.498 \mathrm{e}-15$ & 0 \\
\hline & NFE & $5.0^{*} 10^{4}$ & $5.0 * 10^{4}$ & $5.0^{*} 10^{4}$ & $5.0^{*} 10^{4}$ & $5.0^{*} 10^{4}$ & $5 * 10^{4}$ & $5 * 10^{4}$ \\
\hline \multirow{3}{*}{ Griewank } & Mean & $7.8 \mathrm{e}-04(-)$ & $1.9 \mathrm{e}-05(-)$ & $9.9 \mathrm{e}-08(-)$ & $1.74 \mathrm{e}-07(-)$ & $5.36 \mathrm{e}-13(-)$ & 0 & 0 \\
\hline & SD & $1.2 \mathrm{e}-03$ & $5.8 \mathrm{e}-05$ & $6.0 \mathrm{e}-07$ & $2.33 \mathrm{e}-07$ & $4.77 \mathrm{e}-14$ & 0 & 0 \\
\hline & NFE & $5.0^{*} 10^{4}$ & $5.0^{*} 10^{4}$ & $5.0^{*} 10^{4}$ & $5.0^{*} 10^{4}$ & $5.0^{*} 10^{4}$ & $5 * 10^{4}$ & $5 * 10^{4}$ \\
\hline+ & 6 & 6 & 6 & 6 & 4 & 1 & & \\
\hline- & 0 & 0 & 0 & 0 & 0 & 1 & & \\
\hline$\approx$ & 0 & 0 & 0 & 0 & 2 & 4 & & \\
\hline
\end{tabular}

Table 4 Performance of MTLBO, CABC, GABC, RABC, and IABC

\begin{tabular}{|c|c|c|c|c|c|c|c|}
\hline Function & & $\mathrm{CABC}$ & GABC & $\mathrm{RABC}$ & IABC & TLBO & MTLBO \\
\hline \multirow{3}{*}{ Sphere } & Mean & $2.3 \mathrm{e}-40(-)$ & $3.6 \mathrm{e}-63(-)$ & $9.1 \mathrm{e}-61(-)$ & $5.34 \mathrm{e}-178(-)$ & $4.9 \mathrm{e}-321$ & $4.9 \mathrm{e}-321$ \\
\hline & SD & $1.7 \mathrm{e}-40$ & $5.7 e-63$ & $2.1 \mathrm{e}-60$ & 0 & 0 & 0 \\
\hline & NFE & $1.5^{*} 10^{5}$ & $1.5^{*} 10^{5}$ & $1.5^{*} 10^{5}$ & $1.5^{*} 10^{5}$ & $5^{*} 10^{4}$ & $5 * 10^{4}$ \\
\hline \multirow{3}{*}{ Schwefel 2.22} & Mean & $3.5 \mathrm{e}-30(-)$ & $4.8 \mathrm{e}-45(-)$ & $3.2 \mathrm{e}-74(-)$ & $8.82 \mathrm{e}-127(-)$ & $4.9 e-321$ & $4.9 \mathrm{e}-321$ \\
\hline & $\mathrm{SD}$ & $4.8 \mathrm{e}-30$ & $1.4 \mathrm{e}-45$ & $2.0 \mathrm{e}-73$ & $3.49 \mathrm{e}-126$ & 0 & 0 \\
\hline & NFE & $2.0^{*} 10^{5}$ & $2.0 * 10^{5}$ & $2.0 * 10^{5}$ & $2.0^{*} 10^{5}$ & $5 * 10^{4}$ & $5 * 10^{4}$ \\
\hline \multirow{3}{*}{ Step } & Mean & $0(\sim)$ & $0(\sim)$ & $0(\sim)$ & $0(\sim)$ & 0 & 0 \\
\hline & $\mathrm{SD}$ & 0 & 0 & 0 & 0 & 0 & 0 \\
\hline & NFE & $1.0^{*} 10^{4}$ & $1.0 * 10^{4}$ & $1.0^{*} 10^{4}$ & $1.0 * 10^{4}$ & $5 * 10^{4}$ & $5 * 10^{4}$ \\
\hline \multirow{3}{*}{ Schwefel 1.2} & Mean & $1.3 \mathrm{e}-00(-)$ & $1.5 \mathrm{e}-10(-)$ & $2.3 \mathrm{e}-02(-)$ & $0(\sim)$ & $1 e-323$ & $9.2076 \mathrm{e}-281$ \\
\hline & $\mathrm{SD}$ & $2.7 \mathrm{e}-00$ & $2.7 \mathrm{e}-10$ & 5.1e-01 & 0 & 4.4238 & 0 \\
\hline & NFE & $1.0^{*} 10^{5}$ & $1.0^{*} 10^{5}$ & $1.0^{*} 10^{5}$ & $1.0^{*} 10^{5}$ & $5 * 10^{4}$ & $5 * 10^{4}$ \\
\hline \multirow{3}{*}{ Ackley } & Mean & $1.0 \mathrm{e}-05(-)$ & $1.8 \mathrm{e}-09(-)$ & $9.6 \mathrm{e}-07(-)$ & $3.87 \mathrm{e}-14(-)$ & $4.2633 \mathrm{e}-15$ & $3.5527 \mathrm{e}-15$ \\
\hline & SD & $2.4 \mathrm{e}-06$ & $7.7 e-10$ & $8.3 e-07$ & $8.52 \mathrm{e}-15$ & $1.498 \mathrm{e}-15$ & 0 \\
\hline & NFE & $5.0 * 10^{4}$ & $5.0^{*} 10^{4}$ & $5.0^{*} 10^{4}$ & $5.0^{*} 10^{4}$ & $5 * 10^{4}$ & $5 * 10^{4}$ \\
\hline \multirow{3}{*}{ Griewank } & Mean & $1.2 \mathrm{e}-04(-)$ & $6.0 \mathrm{e}-13(-)$ & $8.7 \mathrm{e}-08(-)$ & $0(\sim)$ & 0 & 0 \\
\hline & $\mathrm{SD}$ & $4.6 \mathrm{e}-04$ & $7.7 \mathrm{e}-13$ & $2.1 \mathrm{e}-08$ & 0 & 0 & 0 \\
\hline & NFE & $5.0^{*} 10^{4}$ & $5.0 * 10^{4}$ & $5.0^{*} 10^{4}$ & $5.0^{*} 10^{4}$ & $5 * 10^{4}$ & $5 * 10^{4}$ \\
\hline+ & 5 & 5 & 5 & 3 & 1 & & \\
\hline - & 0 & 0 & 0 & 0 & 0 & & \\
\hline$\approx$ & 1 & 1 & 1 & 3 & 5 & & \\
\hline
\end{tabular}


Table 5 CEC-2005 Benchmark test functions

\begin{tabular}{|c|c|c|c|c|}
\hline Function & Name & Search range & $\mathrm{D}$ & F_bias \\
\hline$f_{1}$ & Shifted Sphere Function & {$[-100,100]^{D}$} & 10 & -450 \\
\hline$f_{2}$ & Shifted Schwefel's Problem 1.2 & {$[-100,100]^{D}$} & 10 & -450 \\
\hline$f_{3}$ & Shifted Rotated High Conditioned Elliptic Function & {$[-100,100]^{D}$} & 10 & -450 \\
\hline$f_{4}$ & Shifted Schwefel's Problem 1.2 with Noise in Fitness & {$[-100,100]^{D}$} & 10 & -450 \\
\hline$f_{5}$ & Schwefel's Problem 2.6 with Global Optimum on Bounds & {$[-100,100]^{D}$} & 10 & -310 \\
\hline$f_{6}$ & Shifted Rosenbrock's Function & {$[-100,100]^{D}$} & 10 & 390 \\
\hline$f_{7}$ & Shifted Rotated Griewank's Function without Bounds & {$[0,600]^{D}$} & 10 & -180 \\
\hline$f_{8}$ & Shifted Rotated Ackley's Function with Global Optimum on Bounds & {$[-32,32]^{D}$} & 10 & -140 \\
\hline$f_{9}$ & Shifted Rastrigin's Function & {$[-5,5]^{D}$} & 10 & -330 \\
\hline$f_{10}$ & Shifted Rotated Rastrigin's Function & {$[-5,5]^{D}$} & 10 & -330 \\
\hline$f_{11}$ & Shifted Rotated Weierstrass Function & {$[-0.5,0.5]^{D}$} & 10 & 90 \\
\hline$f_{12}$ & Schwefel's Problem 2.13 & {$[-100,100]^{D}$} & 10 & -460 \\
\hline$f_{13}$ & Expanded Extended Griewank's plus Rosenbrock's Function (F8F2) & {$[-3,1]^{D}$} & 10 & -130 \\
\hline$f_{14}$ & Expanded Rotated Extended Scaffe's F6 & {$[-100,100]^{D}$} & 10 & -300 \\
\hline$f_{15}$ & Hybrid Composition Function 1 & {$[-5,5]^{D}$} & 10 & 120 \\
\hline$f_{16}$ & Rotated Hybrid Composition Function 1 & {$[-5,5]^{D}$} & 10 & 120 \\
\hline$f_{17}$ & Rotated Hybrid Composition Function 1 with Noise in Fitness & {$[-5,5]^{D}$} & 10 & 120 \\
\hline$f_{18}$ & Rotated Hybrid Composition Function 2 & {$[-5,5]^{D}$} & 10 & 10 \\
\hline$f_{19}$ & Rotated Hybrid Composition Function 2 with a Narrow Basin for the Global Optimum & {$[-5,5]^{D}$} & 10 & 10 \\
\hline$f_{20}$ & Rotated Hybrid Composition Function 2 with the Global Optimum on the Bounds & {$[-5,5]^{D}$} & 10 & 10 \\
\hline$f_{21}$ & Rotated Hybrid Composition Function 3 & {$[-5,5]^{D}$} & 10 & 360 \\
\hline$f_{22}$ & Rotated Hybrid Composition Function 3 with High Condition Number Matrix & {$[-5,5]^{D}$} & 10 & 360 \\
\hline$f_{23}$ & Non-Continuous Rotated Hybrid Composition Function 3 & {$[-5,5]^{D}$} & 10 & 360 \\
\hline$f_{24}$ & Rotated Hybrid Composition Function 4 & {$[-5,5]^{D}$} & 10 & 260 \\
\hline$f_{25}$ & Rotated Hybrid Composition Function 4 without Bounds & {$[-2,5]^{D}$} & 10 & 260 \\
\hline
\end{tabular}

$f_{\text {cost }}(\{X\})=\left(1+\varepsilon_{1} * \vartheta\right)^{\varepsilon_{2}} * W(\{X\})$,

$\vartheta=\sum_{k=1}^{n} \max \left[0, g_{k}(\{X\})\right]$

where, $W(\{X\}), g_{k}(\{X\})$ and $\vartheta$ are the cost function, the constraint, and the total constraint violation of the optimization problem, respectively. The constants $\varepsilon_{1}$ and $\varepsilon_{2}$ are selected based on the exploration and exploitation rates of the search space. In the present work, $\varepsilon_{1}=1$ and $\varepsilon_{2}$ is changed from 1.5 to 3 .

\subsubsection{Tension/compression spring design}

This problem aims to minimize the weight of the tension/ compression spring shown in Fig. 3. The problem has three design variables including the wire diameter $(d)$, the mean diameter of coil $(D)$, and the number of active coils $(N)$. It is subjected to three nonlinear inequality constraints in terms of shear stress, surge frequency, and deflection and one linear inequality constraint as follows:
Minimze: $f(x)=\left(x_{3}+2\right) x_{2} x_{1}^{2}$

Subject to:

$g_{1}(x)=1-\frac{x_{2}^{3} x_{3}}{71785 x_{1}^{4}} \leq 0$

$g_{2}(x)=\frac{4 x_{2}^{2}-x_{1} x_{2}}{12566\left(x_{2} x_{1}^{3}-x_{1}^{4}\right)}+\frac{1}{5108 x_{1}^{2}}-1 \leq 0$

$g_{3}(x)=1-\frac{140.45 x_{1}}{x_{2}^{2} x_{3}} \leq 0$

$g_{4}(x)=\frac{x_{1}+x_{2}}{1.5}-1 \leq 0$

where

$0.05 \leq x_{1} \leq 2.00,0.25 \leq x_{2} \leq 1.30$, and $2.00 \leq x_{3} \leq 15.00$

The tension/compression spring design problem has been undergone under co-evolutionary DE (CDE) [26], ABC [27], CPSO [28] and HPSO [29]. The convergence histories of the original TLBO and MTLBO for the optimization problem are shown in Fig. 4. 
Table 6 The average error for CEC-2005 benchmark functions

\begin{tabular}{|c|c|c|c|c|c|c|}
\hline & & $f_{1}$ & $f_{2}$ & $f_{3}$ & $f_{4}$ & $f_{5}$ \\
\hline \multirow{2}{*}{ MTLBO } & Mean & $1.0687 \mathrm{e}-13$ & $1.6826 \mathrm{e}-13$ & 124932.8983 & $6.3892 \mathrm{e}-13$ & $1.0186 \mathrm{e}-12$ \\
\hline & SD & $6.4227 \mathrm{e}-14$ & $1.8672 \mathrm{e}-13$ & 108160.5655 & $2.7488 \mathrm{e}-12$ & $1.6671 \mathrm{e}-12$ \\
\hline \multirow{2}{*}{ TLBO } & Mean & $3.2652 \mathrm{e}-10$ & $1.8736 \mathrm{e}-10$ & 83810.8999 & 113.9518 & $2.6713 \mathrm{e}-09$ \\
\hline & SD & $1.346 \mathrm{e}-09$ & $7.7548 \mathrm{e}-10$ & 67935.8848 & 274.4984 & $8.7846 \mathrm{e}-09$ \\
\hline \multirow{3}{*}{ MTLBO } & & $f_{6}$ & $f_{7}$ & $f_{8}$ & $f_{9}$ & $f_{10}$ \\
\hline & Mean & 1.3562 & 1267.056 & 20.3426 & 5.333 & 16.2547 \\
\hline & $\mathrm{SD}$ & 2.0524 & 0.050043 & 0.084342 & 4.2976 & 6.8469 \\
\hline \multirow{2}{*}{ TLBO } & Mean & 908.7199 & 1267.2359 & 20.3597 & 24.7943 & 28.114 \\
\hline & SD & 3099.1562 & 0.29006 & 0.053297 & 8.8988 & 10.5799 \\
\hline \multirow{3}{*}{ MTLBO } & & $f_{11}$ & $f_{12}$ & $f_{13}$ & $f_{14}$ & $f_{15}$ \\
\hline & Mean & 5.2802 & 1009.1863 & 0.62457 & 2.9711 & 285.8865 \\
\hline & SD & 1.0278 & 1656.0728 & 0.25276 & 0.39694 & 188.6135 \\
\hline \multirow{3}{*}{ TLBO } & Mean & 6.1983 & 2498.1546 & 1.219 & 3.0128 & 360.3644 \\
\hline & SD & 1.2389 & 3622.3764 & 0.62644 & 0.37674 & 188.7328 \\
\hline & $f_{1}$ & $f_{16}$ & $f_{17}$ & $f_{18}$ & $f_{19}$ & $f_{20}$ \\
\hline \multirow{2}{*}{ MTLBO } & Mean & 96.6802 & 156.4426 & 855.1239 & 280.7902 & 869.0538 \\
\hline & SD & 418.348 & 62.4886 & 108.6305 & 205.9564 & 1000.5878 \\
\hline \multirow{3}{*}{ TLBO } & Mean & 186.3049 & 171.4382 & 1003.8061 & 993.1936 & 132.8584 \\
\hline & SD & 96.3443 & 37.7033 & 102.8687 & 147.8207 & 97.9305 \\
\hline & & $f_{21}$ & $f_{22}$ & $f_{23}$ & $f_{24}$ & $f_{25}$ \\
\hline \multirow{2}{*}{ MTLBO } & Mean & 138.2112 & 795.7579 & 976.1826 & 248 & 876.1210 \\
\hline & SD & 24.1749 & 43.795 & 227.2545 & 112.2497 & 133.3062 \\
\hline \multirow{2}{*}{ TLBO } & Mean & 183.1771 & 849.2694 & 1210.9344 & 478.4318 & 1027.7150 \\
\hline & $\mathrm{SD}$ & 68.1253 & 97.6388 & 160.8402 & 370.6494 & 97.3425 \\
\hline
\end{tabular}

Table 7 presents the details of the best solutions using the basic TLBO and MTLBO algorithms. The number of population for both TLBO and MTLBO is considered as 10 . Table 8 also compared the statistical results of the considered algorithms with those given by the basic TLBO and MTLBO algorithms. Based on the values of the mean and the standard deviation (SD) inserted in Table 8, it can be found that the MTLBO has outperformed the other algorithm. With far fewer NFE adopted for the TLBO and MTLBO compared to other optimization algorithms, the best results are given for both optimization algorithms. It is worth noting that the performance of MTLBO is slightly better than the TLBO in terms of mean, worst and SD criteria.

\subsubsection{Optimal design of welded beam}

The purpose of the problem is to optimally design a welded beam under certain constraints having minimum cost. The welded beam structure is illustrated in Fig. 5.

As illustrated in the figure, the beam $\mathrm{A}$ is welded to the member B. The optimization problem aims to find the minimum fabrication cost. The design variables are $x_{1}, x_{2}$, $x_{3}, x_{4}$. The constraints of the problem included shear stress $(\tau)$, bending stress of the beam $(\sigma)$, buckling load on the bar $\left(P_{c}\right)$, and the end deflection of the beam $(\delta)$. The optimization problem can be formulated as:

Minimize:

$$
f(x)=1.10471 x_{1}^{2} x_{2}+0.04811 x_{3} x_{4}\left(14.0+x_{2}\right)
$$

Subject to:

$$
\begin{aligned}
& g_{1}(x)=\tau(x)-13600 \leq 0 \\
& g_{2}(x)=\sigma(x)-30000 \leq 0 \\
& g_{3}(x)=x_{x}-x_{4} \leq 0 \\
& g_{4}(x)=\left(0.10471 x_{1}^{2}\right)+0.04811 x_{3} x_{4}\left(14+x_{2}\right)-5.0 \leq 0 \\
& g_{5}(x)=0.125-x_{1} \leq 0 \\
& g_{6}(x)=\delta(x)-0.25 \leq 0 \\
& g_{7}(x)=6000-p_{c}(x) \leq 0
\end{aligned}
$$



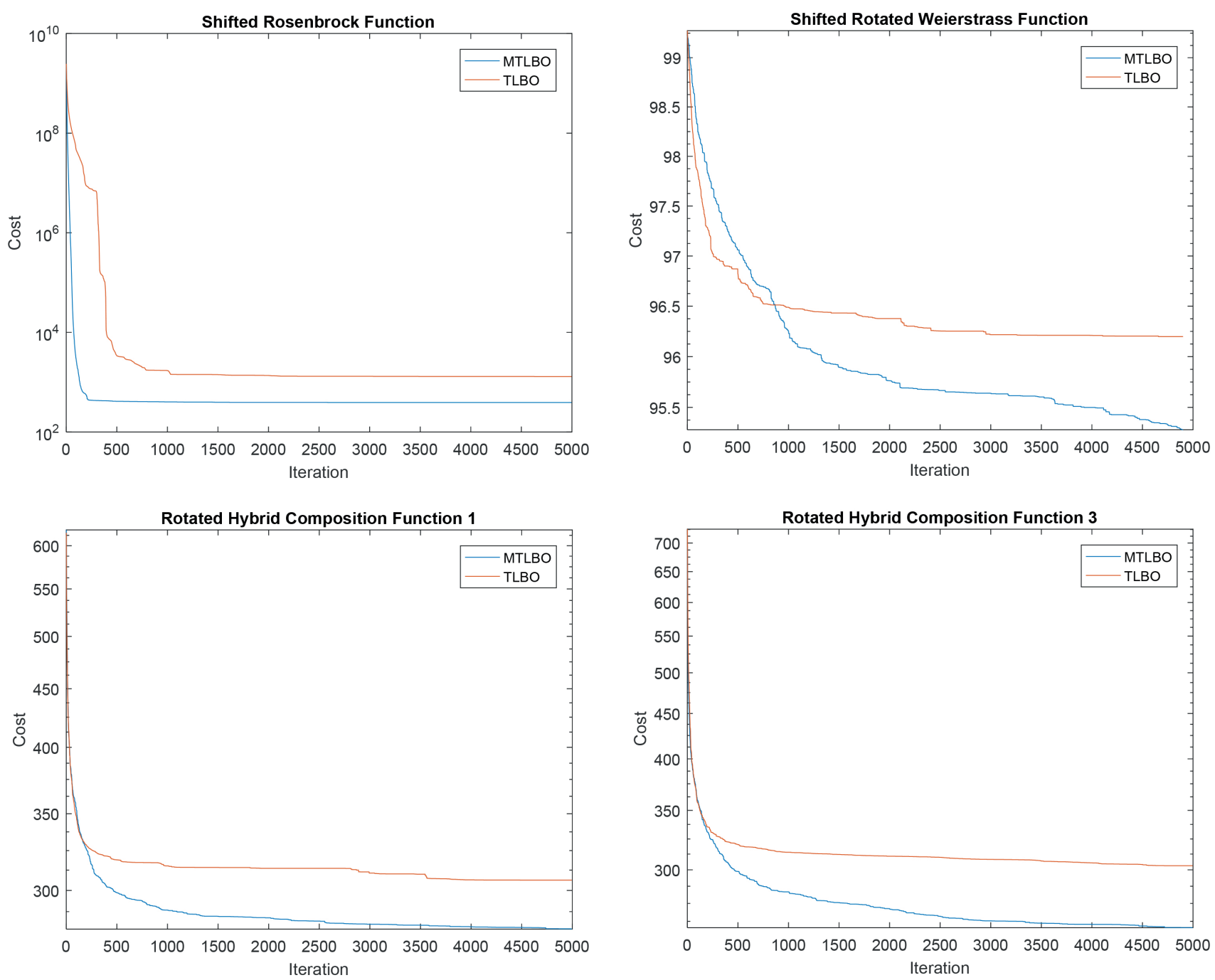

Fig. 2 Convergence rate of the MTLBO and TLBO

where:

$$
\begin{aligned}
& \tau(x)=\sqrt{\left(\tau^{\prime}\right)^{2}+\left(2 \tau^{\prime} \tau^{\prime \prime}\right) \frac{x_{2}}{2 R}+\left(\tau^{\prime \prime}\right)^{2}} \\
& \tau^{\prime}=\frac{6000}{\sqrt{2} x_{1} x_{2}} \\
& \tau^{\prime \prime}=\frac{M R}{J} \\
& M=6000\left(14+\frac{x_{2}}{2}\right) \\
& R=\sqrt{\frac{x_{2}^{2}}{4}+\left(\frac{x_{1}+x_{3}}{2}\right)^{2}} \\
& J=2\left\{x_{1} x_{2} \sqrt{2}\left[\frac{x_{2}^{2}}{12}+\left(\frac{x_{1}+x_{3}}{2}\right)^{2}\right]\right\}
\end{aligned}
$$

$$
\begin{aligned}
& \sigma(x)=\frac{504000}{x_{4} x_{3}^{2}} \\
& \delta(x)=\frac{65856000}{\left(30^{*} 10^{6}\right) x_{4} x_{3}^{3}} \\
& p_{c}(x)=\frac{4.013\left(30 * 10^{6}\right) \sqrt{\frac{x_{3}^{2} x_{4}^{6}}{36}}}{196}\left(1-\frac{x_{3} \sqrt{\frac{30 * 10^{6}}{4\left(12^{*} \cdot 10^{6}\right)}}}{28}\right)
\end{aligned}
$$

$0.1 \leq x_{1}, x_{4} \leq 2.0$, and $0.1 \leq x_{2}, x_{3} \leq 10.0$.

The welded beam optimization design problem has been investigated using the modified differential evolution algorithm (COMDE) [32], ABC [27], hybrid PSO with differential evolution (PSO-DE) [33], co-evolutionary PSO (CPSO) 


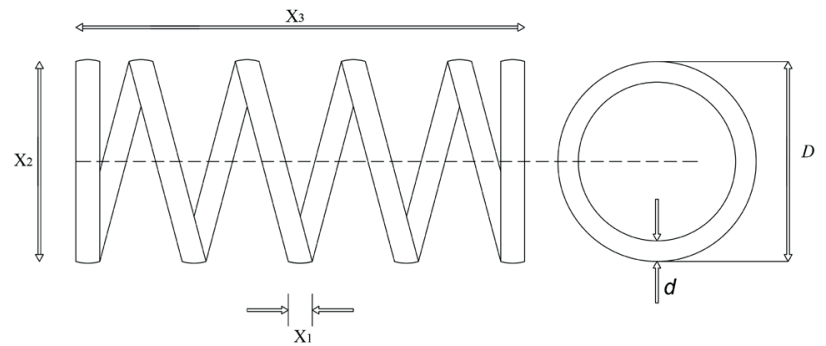

Fig. 3 The tension/compression spring design problem [30]

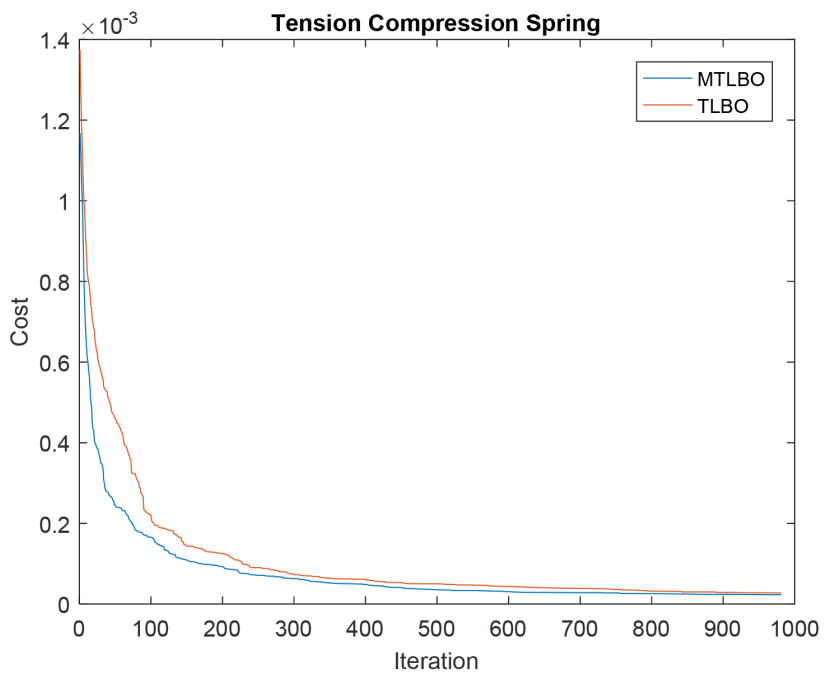

Fig. 4 Convergence graphs for tension/compression spring design problem

[28] and hybrid PSO (HPSO) [29]. Considering the number of population $n P o p=10$ for the basic TLBO and TLBO algorithms, the convergence graphs and optimal parameters of the problem are shown in Fig. 6 and Table 9, respectively. Furthermore, a compression among the statistical results of the considered algorithms with those given by the basic TLBO and MTLBO is indicated in Table 10.

Table 10 confirms the capability of the MTLBO, TLBO, and COMDE to find the optimal solution in all runs. It is evident that the MTLBO has lower values of SD than COMDE, but it has the same mean and NFE with COMDE. Considering a smaller NFE for the MTLBO than the other algorithms, it gives better performance than all other algorithms according to the values of the mean and SD. Also, Fig. 6 shows that the MTLBO converges more rapidly than the original TLBO to the optimal solution.

\subsubsection{A reinforced concrete beam design}

Fig. 7 shows a simplified total cost optimization problem for A 30-ft simple reinforced concrete beam introduced by Amir and Hasegawa [34]. It is subjected to a live load of $2.0 \mathrm{klbf}$ and a dead load (including the weight of the
Table 7 Optimal solutions for the tension/compression spring design problem

\begin{tabular}{lccc}
\hline $\begin{array}{l}\text { Design } \\
\text { variables }\end{array}$ & $x_{1}$ & $x_{2}$ & $x_{3}$ \\
\hline MTLBO & 0.052351 & 0.372865 & 10.407740 \\
TLBO & 0.051565 & 0.353759 & 11.464504 \\
ABC & 0.051749 & 0.358179 & 11.203763 \\
CDE & 0.051609 & 0.354714 & 11.410831 \\
CPSO & 0.051728 & 0.357644 & 11.244543 \\
HPSO & 0.051706 & 0.357126 & 11.265083 \\
\hline
\end{tabular}

Table 8 The statistical results of the tension/compression spring optimization design problem

\begin{tabular}{lccccc}
\hline Method & Best & Mean & Worst & SD & NFE \\
\hline MTLBO & 0.012666 & 0.012686 & 0.012754 & $1.9189 \mathrm{e}-05$ & 20000 \\
TLBO & 0.012665 & 0.012696 & 0.012791 & $2.8982 \mathrm{e}-05$ & 20000 \\
ABC & 0.012665 & 0.012709 & NA & $1.28 \mathrm{e}-02$ & 30000 \\
CDE & 0.0126702 & 0.012703 & 0.012790 & $2.7 \mathrm{e}-05$ & 240000 \\
CPSO & 0.0126747 & 0.012730 & 0.012924 & $5.20 \mathrm{e}-05$ & 200000 \\
HPSO & 0.0126652 & 0.012707 & 0.012719 & $1.58 \mathrm{e}-05$ & 81000 \\
\hline
\end{tabular}

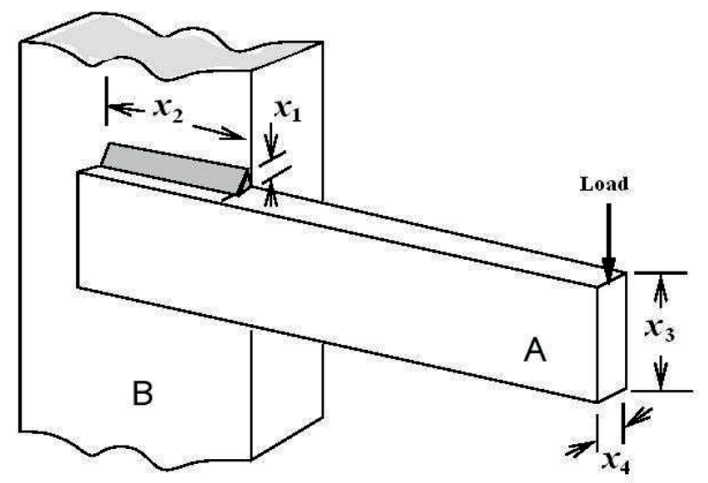

Fig. 5 The optimal design problem of the welded beam [31]

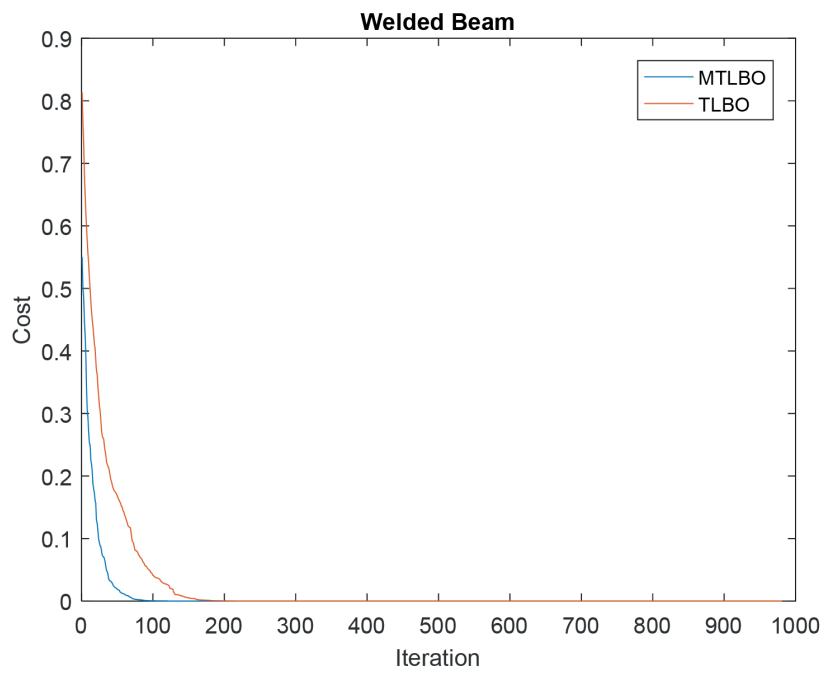

Fig. 6 Convergence graphs for the optimal design of welded beam 
Table 9 Optimal solutions for the welded beam optimization design problem

\begin{tabular}{lcccc}
\hline Design variables & $x_{1}$ & $x_{2}$ & $x_{3}$ & $x_{4}$ \\
\hline MTLBO & 0.2057296 & 3.4704886 & 9.0366239 & 0.2057296 \\
TLBO & 0.2057296 & 3.4704886 & 9.0366239 & 0.2057296 \\
COMDE & NA & NA & NA & NA \\
ABC & 0.20573 & 3.470489 & 9.036624 & 0.20573 \\
PSO-DE & 0.2057296 & 3.4704886 & 9.0366239 & 0.2057296 \\
CPSO & 0.202369 & 3.544214 & 9.04821 & 0.205723 \\
HPSO & 0.20573 & 3.470489 & 9.036624 & 0.20573 \\
\hline
\end{tabular}

NA is used for not available.

Table 10 The statistical results of the welded beam optimization design problem

\begin{tabular}{lccccc}
\hline Method & Best & Mean & Worst & SD & NFE \\
\hline MTLBO & 1.7248523 & 1.7248523 & 1.7248523 & $1.1362 \mathrm{e}-15$ & 20000 \\
TLBO & 1.7248523 & 1.7248523 & 1.7248523 & $2.5007 \mathrm{e}-14$ & 20000 \\
COMDE & 1.7248523 & 1.7248523 & 1.7248523 & $1.60 \mathrm{e}-12$ & 20000 \\
ABC & 1.724852 & 1.741913 & NA & $3.1 \mathrm{e}-02$ & 30000 \\
PSO-DE & 1.724853 & 1.724858 & 1.724881 & $4.1 \mathrm{e}-06$ & 33000 \\
CPSO & 1.728024 & 1.748831 & 1.782143 & $1.29 \mathrm{e}-02$ & 200000 \\
HPSO & 1.724852 & 1.749040 & 1.814295 & $4.00 \mathrm{e}-02$ & 81000 \\
\hline
\end{tabular}

NA is used for not available.

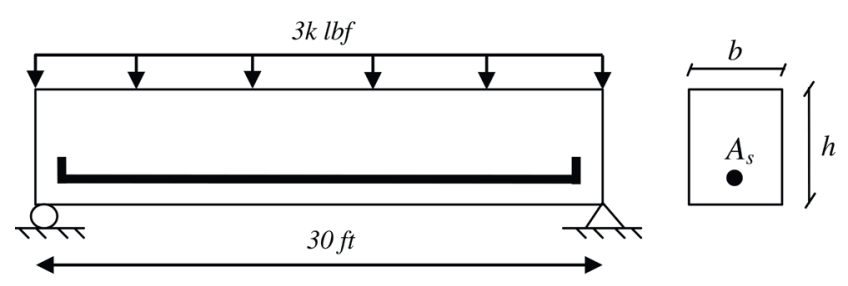

Fig. 7 the reinforced concrete beam design problem [35]

beam) of $1.0 \mathrm{klbf}$. The concrete compressive strength $\left(F_{c}\right)$ and yield stress of the reinforcing steel $\left(F_{y}\right)$ are considered as $5 \mathrm{ksi}$, and $50 \mathrm{ksi}$, respectively. The unit costs of concrete and steel are $\$ 0.02 / \mathrm{in}^{2} /$ linear $\mathrm{ft}$ and $\$ 1.0 / \mathrm{in}^{2} /$ linear $\mathrm{ft}$, respectively. The design variables are the area of the reinforcement $\left(A_{s}\right)$, the width of the beam $(b)$, and the depth of the beam $(h)$. The cross-sectional area of the bar as a discrete variable is selected from the standard bar dimensions reported in [34], while the width of the concrete beam and the depth of the beam are respectively integer and continuous design variables. The effective depth is considered as $0.8 x_{2}$. The structure should meet the required strength according to ACI 318-77 building code as follows:

$$
M_{u}=0.9 A_{s} \sigma_{y}(0.8 h)\left(1.0-0.59 \frac{A_{s} \sigma_{y}}{0.8 b h \sigma_{c}}\right) \geq 1.4 M_{a}+1.7 M_{l}
$$

In which $M_{u}, M_{a}$, and $M_{l}$ are the moments of the beam under the flexural strength, dead load, and live load, respectively. In this case, the values of $M_{d}$ and $M_{l}$ are 1,350 kip-in
2,700 kip-in, respectively. The depth to width ratio is restricted to 4 or less. The optimization problem can be defined as the following formulation:

Minimize: $f\left(A_{s}, b, h\right)=2.9 A_{s}+0.6 b h$

Subject to:

$g_{1}(b, h)=\frac{h}{b}-4 \leq 0$

$g_{2}\left(A_{s}, b, h\right)=180+7.375 \frac{A_{s}^{2}}{b}-A_{s} h \leq 0$.

The variables bound of the cross-sectional area of the reinforcing bar, the width of the beam and the depth of the beam are $\{6.0,6.16,6.32,6.6,7.0,7.11,7.2,7.8,7.9,8.0$, $8.4\} \mathrm{in}^{2},\{28,29,30,31, \ldots, 38,39,40\}$ in and $5 \leq h \leq 10 \mathrm{in}$, respectively. The functions $\mathrm{g}_{1}$ and $\mathrm{g}_{2}$, are the constrained functions derived by Liebman et al. [36].

The problem has been also assessment through Hybrid discrete steepest descent and rotating coordinate directions methods (SD-RC) [34], Generalized Hopfield network-based augmented Lagrange multiplier approach (GHN-ALM) [37], GHN based extended penalty approach (GHN-EP) [37], Adaptive hybrid GA with fuzzy logic controller (FLC-AHGA) [38]. Fig. 8 indicates the convergence graphs for the optimal design of the reinforced concrete beam. Also, Table 11 presents the optimal solutions and the statistical results of the problem by the above-mentioned 


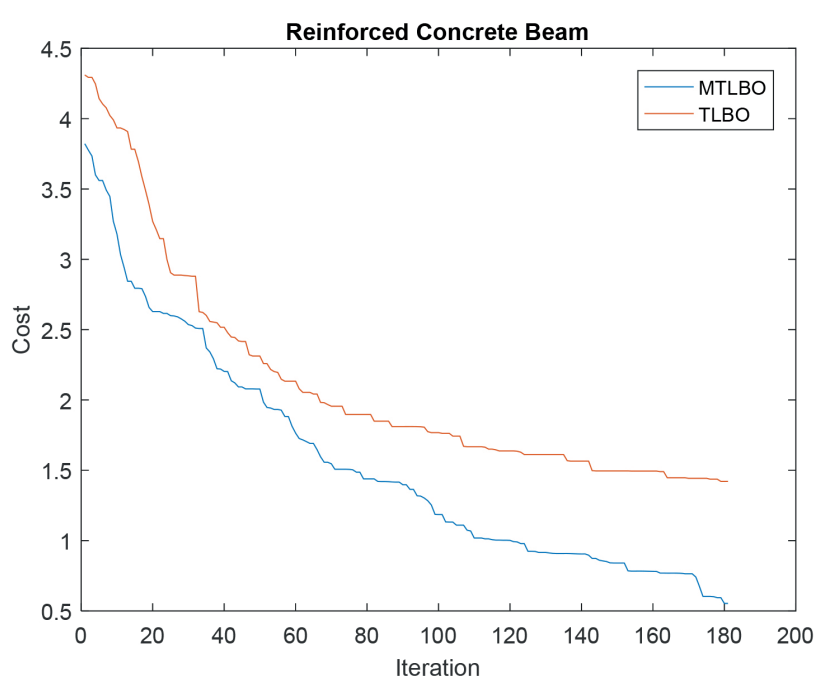

Fig. 8 Convergence graphs for the optimal design of the reinforced

$$
\text { concrete beam }
$$

Table 11 Optimal solutions and statistical results of the reinforced concrete beam design problem

\begin{tabular}{lcccccc}
\hline & \multicolumn{3}{c}{$\begin{array}{c}\text { Optimal solution of the } \\
\text { design variable }\end{array}$} & \multicolumn{3}{c}{ The statistical results } \\
\hline Method & $A_{s}$ & $b$ & $h$ & Mean & SD & NFE \\
MTLBO & 6.32 & 34 & 8.50 & 359.8099 & 1.0511 & 10000 \\
TLBO & 6.6 & 33 & 8.50 & 361.5814 & 1.8981 & 10000 \\
SD-RC & 7.8 & 31 & 7.79 & 374.2 & NA & 10000 \\
GHN-ALM & 6.6 & 33 & 8.495227 & 374.2 & NA & 10000 \\
GHN-EP & 6.32 & 34 & 8.637180 & 362.00648 & NA & 10000 \\
GA & 7.20 & 32 & 8.0451 & 366.1459 & NA & 10000 \\
FLC-AHGA & 6.16 & 35 & 8.7500 & 364.8541 & NA & 10000 \\
\hline NA is
\end{tabular}

NA is used for not available.

optimization algorithms. The number of population for the basic TLBO and MTLBO is adopted as 25. Table 11 shows that the MTLBO with the lowest values of Mean, SD respect to other algorithms, provides an efficient, qualified and robust method to find the optimal design of the reinforced concrete beam.

\subsubsection{Ten-bar truss design using discrete variables}

Another example is the optimal design of a ten-bar truss, shown in Fig. 9. A set of 41 discrete values for possible cross-sectional areas of the members is selected as (1.62, $1.80,1.99,2.13,2.38,2.62,2.88,2.93,3.09,3.13,3.38,3.47$, $3.55,3.63,3.84,3.87,3.88,4.18,4.22,4.49,4.59,4.80,4.97$, 5.12, 5.74, 7.22, 7.97, 11.5, 13.5, 13.9, 14.2, 15.5, 16.0, 16.9, $18.8,19.9,22.0,22.9,26.5,30.0$, and $33.5 \mathrm{in}^{2}$ ). The maximum allowable stress of the truss members is restricted to $\pm 25 \mathrm{ksi}$ while the maximum vertical and horizontal deflection of the nodes is $\pm 2.0 \mathrm{in}$. The unit weight of the material was $0.1 \mathrm{lb} / \mathrm{in}^{3}$ and its elasticity modulus is considered as 107 psi. GA (Mahfouz) [39], GA (Barbosa et al.) [40], ACO (Camp and Bichon) [41], and BB-BC (Camp) [42] has investigated the problem. Table 12 indicates the statistical results of considered algorithms along with details of the best solutions. It is found that the best design is given by the MTLBO algorithm in which the weight of truss was obtained as $5490.75 \mathrm{lb}$. The convergence graphs for the optimal design of the ten-bar truss design are also illustrated in Fig. 10. The mean weight of the best feasible truss designs was $5490.74 \mathrm{lb}$ which is resulted in a standard deviation of $0.13852 \mathrm{lb}$. after 50 runs of the algorithm with the number of a population of 25 . The number of truss analyses needed by the MTLBO algorithm to be converged was 7500. In comparison with GA, ACO, and $\mathrm{BB}-\mathrm{BC}$ algorithms, the MTLBO algorithm requires less computational effort to find the optimal design. For the truss design problem with ten design variables, a comparison between the results given by the MTLBO with those given for other algorithms is remarkable. The standard deviation of the MTLBO is 0.14 , whereas the corresponding values for other algorithms are about 23, 12 and 212, respectively. Therefore, it is concluded that the superiority of the MTLBO is evident for optimization problems with a large number of design variables.

\subsection{Control optimization problems}

Two structural control problems are addressed in this section to validate the proposed MTLBO algorithm. The first problem is the optimal design of TMD as a passive control device for the seismic-excited structure. The second problem is to tune the feedback gain matrix of a controller in a structure equipped with an active tendon system (ATS).

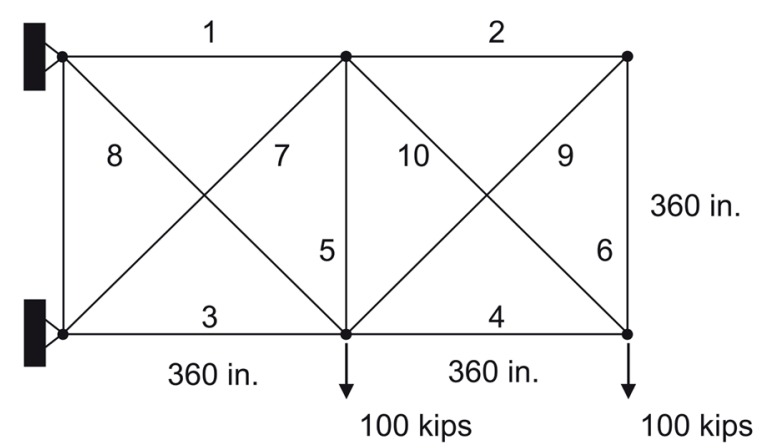

Fig. 9 Ten-bar truss design using discrete variables [43] 
Table 12 The best solutions and the statistical results of the Ten-bar truss design problem

\begin{tabular}{|c|c|c|c|c|c|c|}
\hline Members & GA, Mahfouz [39] & GA, Barbosa et al [40] & ACO, Camp and Bichon [41] & BB-BC, Camp [42] & TLBO & MTLBO \\
\hline 1 & 33.50 & 33.50 & 33.50 & 33.50 & 33.50 & 33.50 \\
\hline 2 & 1.62 & 1.62 & 1.62 & 1.62 & 1.62 & 1.62 \\
\hline 3 & 22.90 & 22.90 & 22.90 & 22.90 & 22.90 & 22.90 \\
\hline 4 & 14.20 & 14.20 & 14.20 & 14.20 & 14.20 & 14.20 \\
\hline 5 & 1.62 & 1.62 & 1.62 & 1.62 & 1.62 & 1.62 \\
\hline 6 & 1.62 & 1.62 & 1.62 & 1.62 & 1.62 & 1.62 \\
\hline 7 & 22.90 & 22.90 & 22.90 & 22.90 & 22.90 & 22.90 \\
\hline 8 & 7.97 & 7.97 & 7.97 & 7.97 & 7.97 & 7.97 \\
\hline 9 & 1.62 & 1.62 & 1.62 & 1.62 & 1.62 & 1.62 \\
\hline 10 & 22.00 & 22.00 & 22.00 & 22.00 & 22.00 & 22.00 \\
\hline Wmin & 5490.74 & 5490.74 & 5490.74 & 5490.74 & 5490.74 & 5490.74 \\
\hline Wavg & NA & 5534.98 & 5510.52 & 5494.17 & 5595.60 & 5490.75 \\
\hline SD & NA & NA & 23.19 & 12.42 & 211.55 & 0.13852 \\
\hline NFE & 8000 & 200000 & 10000 & 8694 & 7500 & 7500 \\
\hline
\end{tabular}

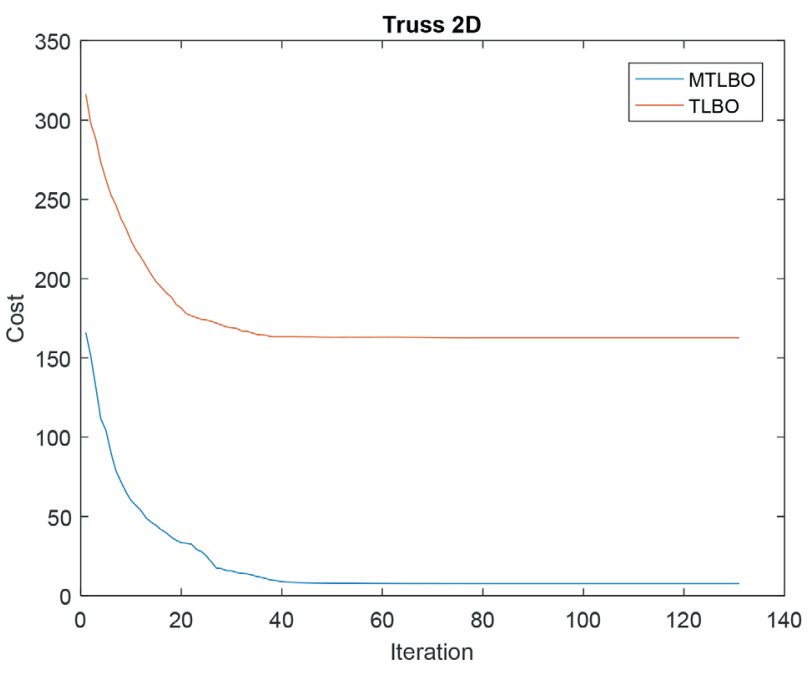

Fig. 10 Convergence graphs for the optimal design of Ten-bar truss design

4.3.1 Optimal design of tuned mass damper parameters The optimal design of a Tuned mass damper (TMD) located on the top story of a ten-story shear building subjected to the artificial earthquake excitation, shown in Fig. 11, is considered for validation of the proposed MTLBO algorithm. The artificial earthquake is produced by a band-limited Gaussian white noise with the following power spectral density function:

$s(\omega)=\left(\frac{4 \xi_{g} \omega_{g} \omega}{\omega^{2}+2 \xi_{g} \omega_{g} \omega+\omega_{g}^{2}}\right)$,

where $\xi_{g}$ and $\omega_{g}$ are the ground damping and frequency, respectively. For numerical simulations, the ground damping and frequency are adopted as 0.3 and $2 \pi \mathrm{rad} / s$, respectively [44].

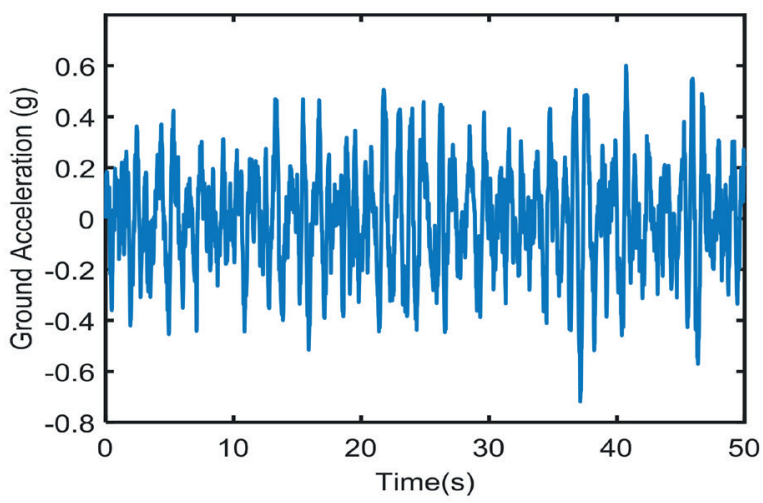

Fig. 11 Time history of the artificial earthquake

The mass, damping coefficient, and stiffness coefficient of each story are $360 \mathrm{ton}, 6.2 \mathrm{MNs} / \mathrm{m}$, and $650 \mathrm{MN} / \mathrm{m}$, respectively. The optimization problem aims to find the optimal TMD parameters, including TMD mass $\left(m_{d}\right)$, damping coefficient $\left(c_{d}\right)$ and stiffness coefficient $\left(k_{d}\right)$, for minimizing the maximum top story displacement as follows:

Minimize $I=\frac{\max _{t}\|x(t)\|}{\max _{t}\|\tilde{x}(t)\|}$

Subject to:

$m_{d} \leq 108, c_{d} \leq 1000$, and $k_{d} \leq 5000$

$\max _{t}\left\|x_{T M D}(t)-x_{\text {top }}(t)\right\| \ll 150 \mathrm{~cm}$,

where $I$ is the maximum floor displacement of the structure with the TMD normalized to the corresponding response for the structure without TMD. A limitation for the maximum TMD stroke is added to the problem as a constrained. In other words, the term of $\max _{t}\left\|x_{T M D}(t)-x_{\text {top }}(t)\right\|$ refers to 
the maximum relative TMD displacement respect to the maximum roof displacement which should be less than $150 \mathrm{~cm}$. Using a penalty method, the complicated constrained optimization problem can be converted to an unconstrained optimization problem as follows:

$$
\begin{aligned}
& J=I \times(1+50 \max ([0, \alpha])) \\
& \alpha=\frac{\max _{t}\left\|x_{T M D}(t)-x_{\text {Roof }}(t)\right\|}{150}-1 .
\end{aligned}
$$

For a population number of 10 , the mean, best, worst, and SD results for 30 runs are inserted in Table 13. The convergence graphs for both TLBO and MTLBO are illustrated in Fig. 12. Furthermore, the optimal parameters of the TMD using the TLBO and MTLBO are inserted in Table 14. Some optimal design scenarios for the mentioned problem reported by GA in [7], Lee et al. [45] and charged system search (CSS) in [9], are also inserted in the Table for comparison purposes.

The maximum seismic responses of the structure subjected to the El Centro (1940) NS earthquake in terms of maximum floor displacement and acceleration are shown in Tables 15 and 16, respectively. The optimized TMD by TLBO and MTLBO gives better results than GA and the results given by Lee et al. in the reduction of structural responses in terms of floor displacement and acceleration. An average reduction of $37.09 \%$ in the maximum

Table 13 The statistical results of the optimal design problem of TMD

\begin{tabular}{lcccc}
\hline Method & Mean & Best & Worst & SD \\
\hline TLBO & 0.484426 & 0.483998 & 0.488119 & $9.227553 \mathrm{e}-04$ \\
MTLBO & 0.484277 & 0.483996 & 0.485167 & $2.763895 \mathrm{e}-04$ \\
\hline
\end{tabular}

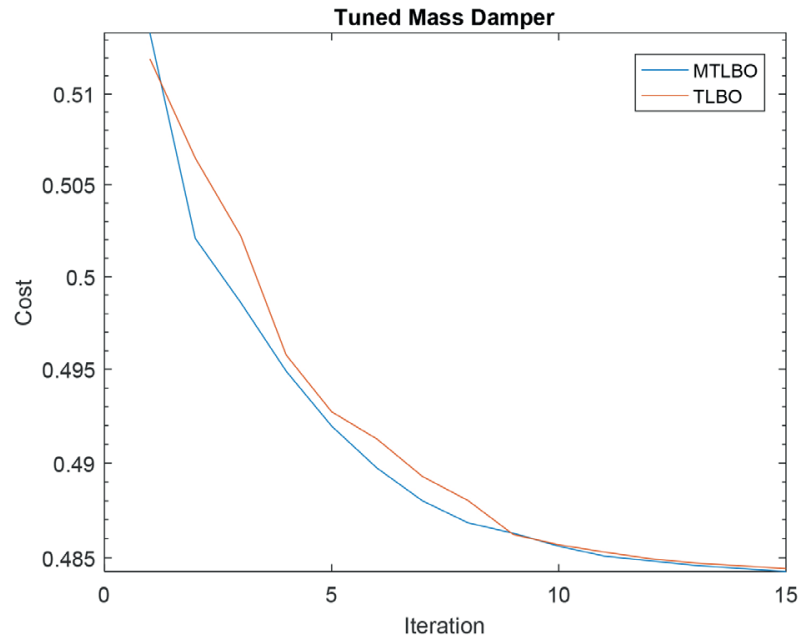

Fig. 12 Convergence graphs for the optimal design of TMD

Table 14 Optimum TMD parameters for the optimal design problem of TMD

\begin{tabular}{lccc}
\hline \multirow{2}{*}{ Methods } & \multicolumn{3}{c}{ Optimum parameters } \\
& $m_{d}$ (ton) & $c_{d}(\mathrm{kNs} / \mathrm{m})$ & $k_{d}(\mathrm{kN} / \mathrm{m})$ \\
\hline GA [7] & 108 & 151.5 & 3750 \\
Lee et al. [45] & 108 & 271.79 & 4126.93 \\
CSS [9] & 108 & 88.697 & 4207.735 \\
TLBO & 107.99 & 214.81 & 4119.55 \\
MTLBO & 108 & 214.82 & 4119.83 \\
\hline
\end{tabular}

displacement and an average reduction of $29.44 \%$ in the maximum acceleration of floors are obtained for both TLBO and MTLBO. However, as found from Table 13, the MTLBO with a less SD provides reliable results than the TLBO. The MTLOB performs better than CSS in the reduction of the maximum floor acceleration, while the CSS results in more reduction in the maximum floor

Table 15 Maximum displacements of stories subjected to the El Centro (1940) NS earthquake

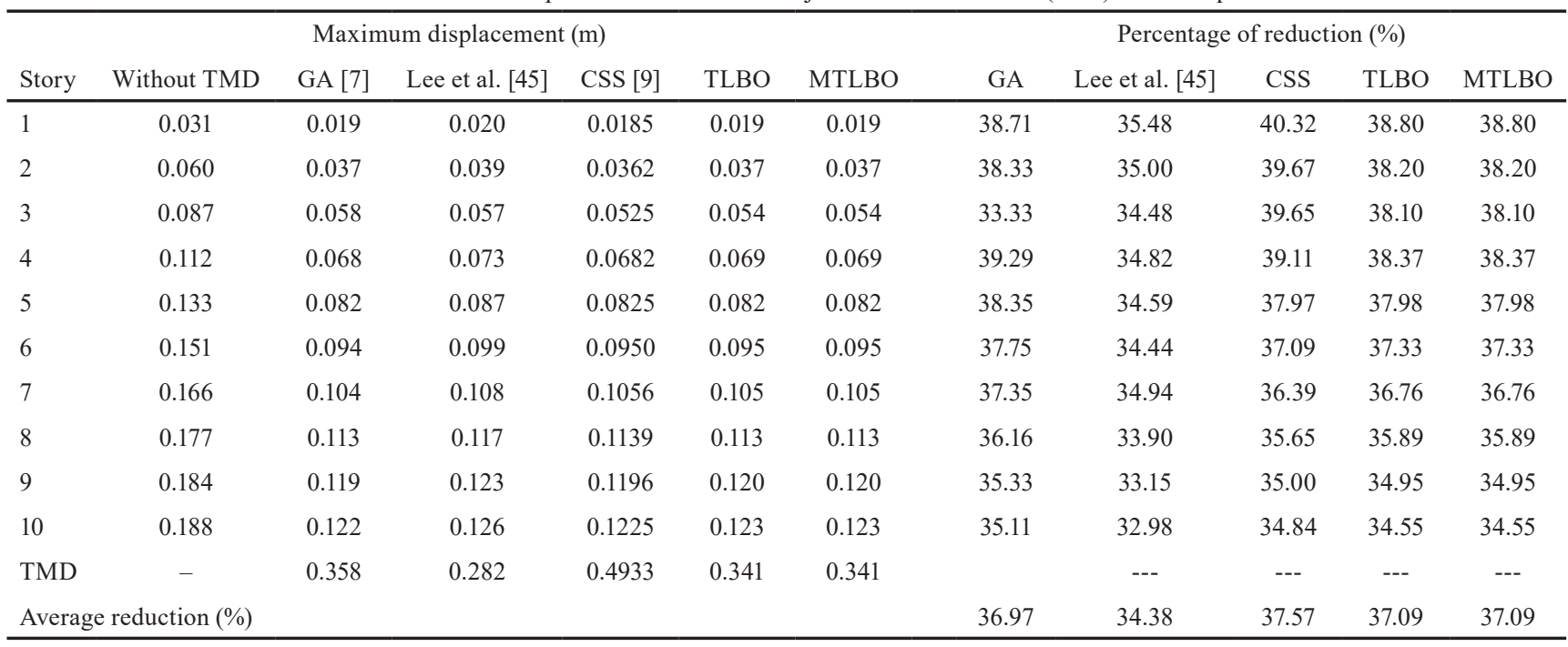


Table 16 Maximum accelerations of stories subjected to the El Centro (1940) NS earthquake

\begin{tabular}{|c|c|c|c|c|c|c|c|c|c|c|c|}
\hline \multirow[b]{2}{*}{ Story } & \multicolumn{6}{|c|}{ Maximum acceleration $\left(\mathrm{m} / \mathrm{s}^{2}\right)$} & \multicolumn{5}{|c|}{ Percentage of reduction $(\%)$} \\
\hline & Without TMD & GA [7] & Lee et al. [45] & CSS [9] & TLBO & MTLBO & GA & Lee et al. [45] & CSS & TLBO & MTLBO \\
\hline 1 & 2.89 & 2.70 & 2.67 & 3.4260 & 2.66 & 2.66 & 6.57 & 7.61 & 1.12 & 8.03 & 8.03 \\
\hline 2 & 3.97 & 3.03 & 3.10 & 5.2593 & 3.02 & 3.02 & 23.68 & 21.91 & 1.47 & 24.01 & 24.01 \\
\hline 3 & 4.93 & 3.53 & 3.64 & 5.9736 & 3.51 & 3.51 & 28.40 & 26.17 & 2.60 & 28.90 & 28.90 \\
\hline 4 & 5.68 & 3.94 & 3.99 & 6.2626 & 3.88 & 3.88 & 30.63 & 29.75 & 14.56 & 31.75 & 31.75 \\
\hline 5 & 6.14 & 4.08 & 4.12 & 6.3223 & 4.02 & 4.02 & 33.55 & 32.90 & 23.59 & 34.57 & 34.57 \\
\hline 6 & 6.55 & 3.83 & 4.14 & 6.1456 & 3.88 & 3.88 & 41.53 & 36.79 & 30.00 & 40.81 & 40.81 \\
\hline 7 & 6.71 & 4.39 & 4.26 & 5.7233 & 4.30 & 4.30 & 34.58 & 36.51 & 36.77 & 35.97 & 35.97 \\
\hline 8 & 7.01 & 5.05 & 4.95 & 5.5797 & 4.99 & 4.99 & 27.96 & 29.39 & 39.72 & 28.82 & 28.83 \\
\hline 9 & 7.84 & 5.53 & 5.44 & 5.8272 & 5.46 & 5.46 & 29.46 & 30.61 & 38.19 & 30.38 & 30.39 \\
\hline 10 & 8.32 & 5.81 & 5.72 & 5.9564 & 5.73 & 5.73 & 30.17 & 31.25 & 37.62 & 31.13 & 31.13 \\
\hline \multicolumn{7}{|c|}{ Average reduction (\%) } & 28.65 & 28.29 & 22.56 & 29.44 & 29.44 \\
\hline
\end{tabular}

displacement. It is noted that the results of the CSS are obtained for an unconstraint TMD design problem (without the TMD stroke constraint defined in Eq. (13). In other words, the optimal design of the TMD given by CSS does not satisfy the TMD stroke constraint during the artificial earthquake excitation.

\subsubsection{Optimal active control design}

A three-story building model studied in [46] is considered. An ATS is installed between the first floor and the ground floor. The mass, damping, and stiffness matrices of the model are as follows:

$$
\begin{aligned}
& \boldsymbol{M}_{s}=\left[\begin{array}{ccc}
98.3 & 0 & 0 \\
0 & 98.3 & 0 \\
0 & 0 & 98.3
\end{array}\right](\mathrm{kg}), \\
& \boldsymbol{C}_{s}=\left[\begin{array}{ccc}
175 & -50 & 0 \\
-50 & 100 & -50 \\
0 & -50 & 50
\end{array}\right](N . \mathrm{sec} / \mathrm{m}), \\
& \boldsymbol{K}_{s}=10^{5}\left[\begin{array}{ccc}
12.0 & -6.84 & 0 \\
-6.84 & 13.7 & -6.84 \\
0 & -6.84 & 6.84
\end{array}\right]\left(\frac{N}{m}\right) .
\end{aligned}
$$

Considering $\boldsymbol{Z}(t)=\left[x_{1} x_{2} x_{3} \dot{x}_{1} \dot{x}_{2} \dot{x}_{3}\right]^{T}, \boldsymbol{L}$ and $\boldsymbol{H}$ as the location vectors of the control force and ground acceleration, the state space form of the equation of motion of the structural model can be stated as:

$$
\dot{Z}(t)=\boldsymbol{A Z}(\boldsymbol{t})+\boldsymbol{B} u(t)+\boldsymbol{H} \ddot{x}_{g}(t)
$$

$$
\boldsymbol{A}=\left[\begin{array}{cc}
\mathbf{0}_{3 \times 3} & \boldsymbol{I}_{3 \times 3} \\
-\boldsymbol{M}_{s}^{-1} \boldsymbol{K}_{s} & -\boldsymbol{M}_{s}^{-1} \boldsymbol{C}_{s}
\end{array}\right], \boldsymbol{B}=\left[\mathbf{0}_{3 \times 1}-\boldsymbol{M}_{s}^{-1} \boldsymbol{L}\right]^{T}
$$

Considering the cost function defined in Eq. (15), the active control force of the linear-quadratic regulator (LQR) controller is given by Eq. (16).

$$
\begin{aligned}
& J=\int_{0}^{t}\left\{\boldsymbol{Z}(t)^{T} \boldsymbol{Q Z}(t)+\boldsymbol{R}^{T} \boldsymbol{u}(t) \boldsymbol{R}\right\} d t, \\
& u(t)=-\boldsymbol{G} \boldsymbol{Z}(t)=-\boldsymbol{R}^{-1} \boldsymbol{B}^{T} \boldsymbol{P Z}(t),
\end{aligned}
$$

where $\boldsymbol{G}$ is a feedback gain matrix of the controller. Also, $\boldsymbol{Q}$ and $\boldsymbol{R}$ are the symmetric weighting matrices. Furthermore, $\boldsymbol{P}$ is a semi-positive definite matrix that can be obtained from the following Riccati equation:

$$
\boldsymbol{P} \boldsymbol{A}+\boldsymbol{A}^{T} \boldsymbol{P}-\boldsymbol{P B} \boldsymbol{R}^{-1} \boldsymbol{B}^{T} \boldsymbol{P}+\boldsymbol{Q}=0 .
$$

In the LQR controller, the weighting matrices $\boldsymbol{Q}$ and $\boldsymbol{R}$ are the design matrices for optimal tuning of the control force. For the problem, the weighting matrices are assumed as the following form:

$$
\boldsymbol{Q}=\operatorname{diag}\left(\left[\begin{array}{llllll}
\alpha & 0 & 0 & 1 & 1 & 1
\end{array}\right]\right), \boldsymbol{R}=10^{-\beta} .
$$

The maximum displacement of floors in the controlled structure normalized to the corresponding response in the uncontrolled structure is assumed as a cost function to tune the optimal design variables i.e. $\alpha$ and $\beta$. The TLBO and MTLBO algorithms are utilized for optimal tuning of the weighting matrices. For a population of 10, the optimal design and statistical results of the active control design problem for 30 runs are shown in Table 17. The convergence graphs for the problem are displayed in Fig. 13. Similar results are obtained for both algorithms, while the MTLBO algorithm resulted in a smaller SD and a higher convergence than rate than the TLBO algorithm. Time histories of the top floor displacement, top floor acceleration, 
The optimal design and statistical results of the active control design problem

\begin{tabular}{llccccc}
\hline Method & $\alpha$ & $\beta$ & Mean & Best & Worst & SD \\
\hline MTLBO & 1 & 7.475728 & 0.234946 & 0.234946 & 0.234946 & $7.432602 \mathrm{e}-14$ \\
\hline TLBO & 1 & 7.476714 & 0.234946 & 0.234946 & 0.234954 & $1.674994 \mathrm{e}-06$ \\
\hline
\end{tabular}

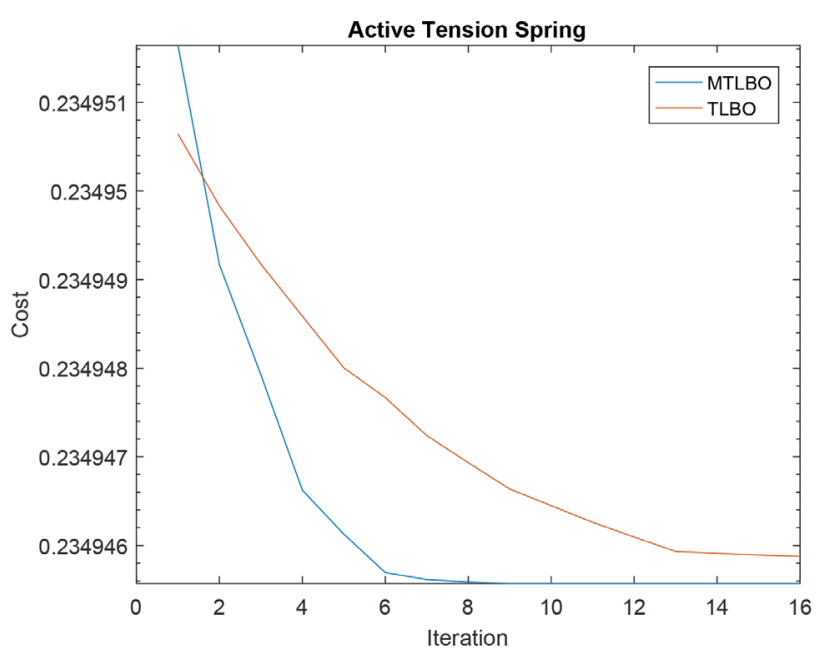

Fig. 13 Convergence graphs for the optimal design of TMD

first-floor drift, and control force of the structure subjected to the scaled $1940 \mathrm{El}$ Centro and 1995 Kobe earthquakes are displayed in Figs. 14 and 15, respectively. A significant reduction is shown in the seismic responses of the structure equipped with ATS in comparison with the uncontrolled ones. The similar results are obtained for the TLBO and MTLBO algorithms.

\section{Conclusions}

The design problems of the passive and active control devices for the seismic-excited structures can be defined as some of the optimization problems that demand simple, accurate, and fast optimization algorithms. For this purpose, a new modified TLBO algorithm, namely MTLBO, is proposed here. In the MTLBO, an extra term was added to the basic TLBO in the both teacher and learner phases. The performance of MTLBO was firstly validated for some unconstrained and constrained engineering benchmarks. To compare the efficiency of the MTLBO, some evolutionary optimization techniques such as PSO, DE, $\mathrm{GA}$, and $\mathrm{ABC}$ along with their various variants were considered. Considering the statistical results undertaken for assessing the performance and reliability of optimization algorithms, it was concluded that the MTLBO was able to give reliable and better results than other algorithms. Furthermore, it was found that the superiority of the MTLBO was evident for optimization problems with a large number of design variables. For some optimization problems, the MTLBO also resulted in the best solution with less function evaluation numbers and computational
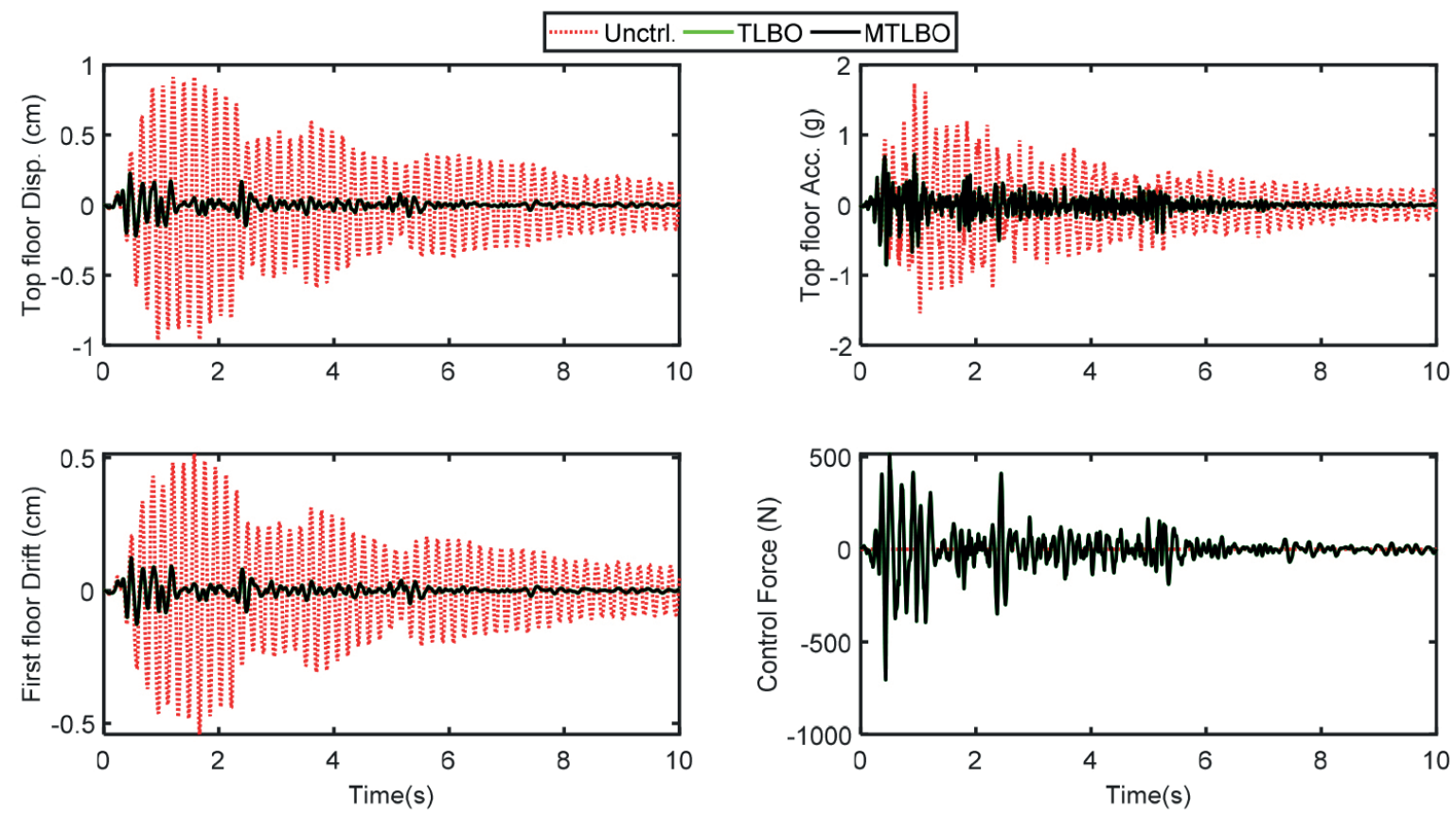

Fig. 14 Time histories of the top floor displacement, top floor acceleration, first-floor drift and control force of the structure subjected to the El 

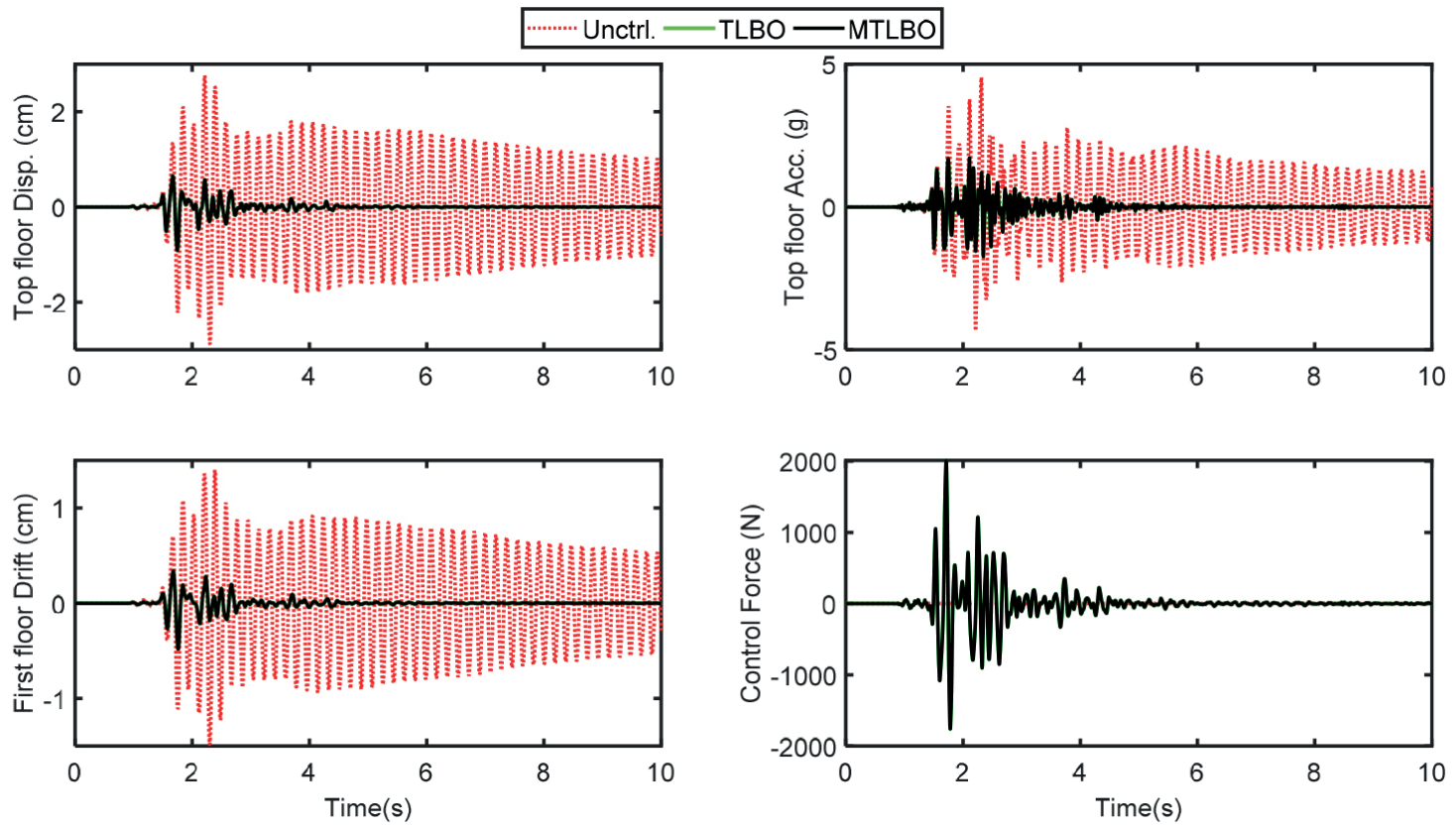

Fig. 15 Time histories of the top floor displacement, top floor acceleration, first-floor drift and control force of the structure subjected to the Kobe earthquake

efforts rather than other algorithms. Finally, the efficiency of the MTLBO was investigated for the optimal tuning of the parameters in structural engineering problems consisting of passive and active control problems. It was

\section{References}

[1] Etedali, S., Akbari, M., Seifi, M. "MOCS-based optimum design of TMD and FTMD for tall buildings under near-field earthquakes including SSI effects", Soil Dynamics and Earthquake Engineering, 119, pp. 36-50, 2019.

https://doi.org/10.1016/j.soildyn.2018.12.027

[2] Fahimi Farzam, M., Kaveh, A. "Optimum Design of Tuned Mass Dampers Using Colliding Bodies Optimization in Frequency Domain", Iranian Journal of Science and Technology, Transactions of Civil Engineering, 44, pp. 787-802, 2020.

https://doi.org/10.1007/s40996-019-00296-6

[3] Ghasemi, M. R., Shabakhty, N., Enferadi, M. H. "Optimized SMA Dampers in Vibration Control of Jacket-type Offshore Structures (Regular Waves)", International Journal of Coastal and Offshore Engineering, 2(4), pp. 25-35, 2019.

https://doi.org/10.29252/ijcoe.2.4.25

[4] Jarrahi, H., Asadi, A., Khatibinia, M., Etedali, S. "Optimal design of rotational friction dampers for improving seismic performance of inelastic structures", Journal of Building Engineering, 27, Article number: 100960, 2020.

https://doi.org/10.1016/j.jobe.2019.100960

[5] Kaveh, A., Farzam, M. F., Maroofiazar, R. "Comparing $\mathrm{H}_{2}$ and $\mathrm{H}_{\infty}$ Algorithms for Optimum Design of Tuned Mass Dampers under Near-Fault and Far-Fault Earthquake Motions", Periodica Polytechnica Civil Engineering, 64(3), pp. 828-844, 2020 https://doi.org/10.3311/PPci.16389 found that the MTLBO algorithm resulted in faster and better performance than the basic TLBO. Consequently, the MTLBO can be successfully extended and utilized for particular engineering optimization problems.

[6] Kaveh, A., Fahimi Farzam, M., Hojat Jalali, H., Maroofiazar, R. "Robust optimum design of a tuned mass damper inerter", Acta Mechanica, 231, pp. 3871-3896, 2020. https://doi.org/10.1007/s00707-020-02720-9

[7] Hadi, M. N. S., Arfiadi, Y. "Optimum Design of Absorber for MDOF Structures", Journal of Structural Engineering, 124(11), pp. 12721280,1998

https://doi.org/10.1061/(ASCE)0733-9445(1998)124:11(1272)

[8] Ghasemi, M. R., Varaee, H. "Damping vibration-based IGMM optimization algorithm: fast and significant", Soft Computing, 23, pp. 451-481, 2019.

https://doi.org/10.1007/s00500-017-2804-3

[9] Kaveh, A., Mohammadi, S., Khadem Hosseini, O., Keyhani, A., Kalatjari, V. R. "Optimum parameters of tuned mass dampers for seismic applications using charged system search", Iranian Journal of Science and Technology, Transactions of Civil Engineering, 39(C1), pp. 21-40, 2015. https://doi.org/10.22099/IJSTC.2015.2739

[10] Etedali, S., Zamani, A.-A., Tavakoli, S. "A GBMO-based PI'D ${ }^{\mu}$ controller for vibration mitigation of seismic-excited structures", Automation in Construction, 87, pp. 1-12, 2018. https://doi.org/10.1016/j.autcon.2017.12.005

[11] Goldberg, D. E. "Genetic Algorithms in Search, Optimization, and Machine Learning", Addison-Wesley Publishing, Boston, MA, USA, 1989 
[12] Kennedy, J., Eberhart, R. "Particle swarm optimization", In: Proceedings of ICNN'95 - International Conference on Neural Networks, Perth, WA, Australia, 1995, pp. 1942-1948. https://doi.org/10.1109/ICNN.1995.488968

[13] Shabani, A., Asgarian, B., Salido, M., Asil Gharebaghi, S. "Search and rescue optimization algorithm: A new optimization method for solving constrained engineering optimization problems", Expert Systems with Applications, 161, Article number: 113698, 2020. https://doi.org/10.1016/j.eswa.2020.113698

[14] Varaee, H., Ghasemi, M. R. "Engineering optimization based on ideal gas molecular movement algorithm", Engineering with Computers, 33, pp. 71-93, 2017. https://doi.org/10.1007/s00366-016-0457-y

[15] Nobahari, M., Ghasemi, M. R., Shabakhty, N. "A novel heuristic search algorithm for optimization with application to structural damage identification", Smart Structures and Systems, 19(4), pp. 449-461, 2017. https://doi.org/10.12989/sss.2017.19.4.449

[16] Kaveh, A., Kooshkbaghi, M. "Enhanced Artificial Coronary Circulation System Algorithm for Truss Optimization with Multiple Natural Frequency Constraints", Periodica Polytechnica Civil Engineering, 63(2), pp. 362-376, 2019.

https://doi.org/10.3311/PPci.13562

[17] Moez, H., Kaveh, A., Taghizadieh, N. "Natural Forest Regeneration Algorithm for Optimum Design of Truss Structures with Continuous and Discrete Variables", Periodica Polytechnica Civil Engineering, 60(2), pp. 257-267, 2016.

https://doi.org/10.3311/PPci.8884

[18] Kaveh, A., Talatahari, S., Khodadadi, N. "The Hybrid Invasive Weed Optimization-Shuffled Frog-leaping Algorithm Applied to Optimal Design of Frame Structures", Periodica Polytechnica Civil Engineering, 63(3), pp. 882-897, 2019. https://doi.org/10.3311/PPci.14576

[19] Arzani, H., Kaveh, A., Kamalinejad, M. "Optimal Design of Pitched Roof Rigid Frames with Non-Prismatic Members Using Quantum Evolutionary Algorithm", Periodica Polytechnica Civil Engineering, 63(2), pp. 593-607, 2019. https://doi.org/10.3311/PPci.14091

[20] Shabani, A., Asgarian, B., Salido, M. "Search and Rescue Optimization Algorithm for Size Optimization of Truss Structures with Discrete Variables", Numerical Methods in Civil Engineering, 3(3), pp. 28-39, 2019. [online] Available at: http://nmce.kntu.ac.ir/ article-1-197-en.html

[21] Rao, R. V., Savsani, V. J., Vakharia, D. P. "Teaching-LearningBased Optimization: An optimization method for continuous non-linear large scale problems", Information Sciences, 183(1), pp. $1-15,2012$. https://doi.org/10.1016/j.ins.2011.08.006

[22] Rao, R. V., Savsani, V. J., Vakharia, D. P. "Teaching-learning-based optimization: A novel method for constrained mechanical design optimization problems", Computer-Aided Design, 43(3), pp. 303$315,2011$.

https://doi.org/10.1016/j.cad.2010.12.015
[23] Nayak, J., Naik, B., Kanungo, D. P., Behera, H. S. "A hybrid elicit teaching learning based optimization with fuzzy c-means (ETLBOFCM) algorithm for data clustering", Ain Shams Engineering Journal, 9(3), pp. 379-393, 2018. https://doi.org/10.1016/j.asej.2016.01.010

[24] Dang, M. P., Le, H. G., Chau, N. L., Dao, T.-P. "A multi-objective optimization design for a new linear compliant mechanism", Optimization and Engineering, 21, pp. 673-705, 2020. https://doi.org/10.1007/s11081-019-09469-8

[25] Satapathy, S. C., Naik, A. "Modified Teaching-Learning-Based Optimization algorithm for global numerical optimization -A comparative study", Swarm and Evolutionary Computation, 16, pp. 28-37, 2014. https://doi.org/10.1016/j.swevo.2013.12.005

[26] Huang, F., Wang, L., He, Q. "An effective co-evolutionary differential evolution for constrained optimization", Applied Mathematics and Computation, 186(1), pp. 340-356, 2007. https://doi.org/10.1016/j.amc.2006.07.105

[27] Akay, B., Karaboga, D. "Artificial bee colony algorithm for largescale problems and engineering design optimization", Journal of Intelligent Manufacturing, 23, pp. 1001-1014, 2012. https://doi.org/10.1007/s10845-010-0393-4

[28] He, Q., Wang, L. "A hybrid particle swarm optimization with a feasibility-based rule for constrained optimization", Applied Mathematics and Computation, 186(2), pp. 1407-1422, 2007. https://doi.org/10.1016/j.amc.2006.07.134

[29] He, Q., Wang, L. "An effective co-evolutionary particle swarm optimization for constrained engineering design problems", Engineering Applications of Artificial Intelligence, 20(1), pp. 89-99, 2007. https://doi.org/10.1016/j.engappai.2006.03.003

[30] Li, G., Shuang, F., Zhao, P., Le, C. "An Improved Butterfly Optimization Algorithm for Engineering Design Problems Using the Cross-Entropy Method", Symmetry, 11(8), Article number: 1049, 2019.

https://doi.org/10.3390/sym11081049

[31] Cagnina, L. C., Esquivel, S. C., Coello, C. A. C. "Solving engineering optimization problems with the simple constrained particle swarm optimizer", Informatica, 32(3), pp. 319-326, 2008. [online] Available at: http://www.informatica.si/index.php/informatica/ article/view/204

[32] Mohamed, A. W., Sabry, H. Z. "Constrained optimization based on modified differential evolution algorithm", Information Sciences, 194, pp. 171-208, 2012. https://doi.org/10.1016/j.ins.2012.01.008

[33] Liu, H., Cai, Z., Wang, Y. "Hybridizing particle swarm optimization with differential evolution for constrained numerical and engineering optimization", Applied Soft Computing, 10(2), pp. 629-640, 2010.

https://doi.org/10.1016/j.asoc.2009.08.031

[34] Amir, H. M., Hasegawa, T. "Nonlinear Mixed-Discrete Structural Optimization", Journal of Structural Engineering, 115(3), pp. 626646, 1989. https://doi.org/10.1061/(ASCE)0733-9445(1989)115:3(626) 
[35] Gandomi, A. H., Yang, X.-S., Alavi, A. H. "Cuckoo search algorithm: a metaheuristic approach to solve structural optimization problems", Engineering with Computers, 29, pp. 17-35, 2013. https://doi.org/10.1007/s00366-011-0241-y

[36] Liebman, J. S., Chanaratna, V., Khachaturian, N. "Discrete structural optimization", Journal of the Structural Division, 107(11), pp. 2177-2197, 1981. [online] Available at: https://cedb.asce.org/ CEDBsearch/record.jsp?dockey $=0010542$

[37] Shih, C. J., Yang, Y. C. "Generalized Hopfield network based structural optimization using sequential unconstrained minimization technique with additional penalty strategy", Advances in Engineering Software, 33(7-10), pp. 721-729, 2002. https://doi.org/10.1016/S0965-9978(02)00060-1

[38] Yun, Y. "Study on Adaptive Hybrid Genetic Algorithm and Its Applications to Engineering Design Problems", PhD Dissertation, Waseda University, 2005.

[39] Mahfouz, S. Y. "Design optimization of structural steelwork", PhD Dissertation, University of Bradford, 1999. [online] Available at: https://ethos.bl.uk/OrderDetails.do?uin=uk.bl.ethos. 534650

[40] Barbosa, H. J. C., Lemonge, A. C. C., Borges, C. C. H. "A genetic algorithm encoding for cardinality constraints and automatic variable linking in structural optimization", Engineering Structures, 30(12), pp. 3708-3723, 2008.

https://doi.org/10.1016/j.engstruct.2008.06.014
[41] Camp, C. V., Bichon, B. J. "Design of Space Trusses Using Ant Colony Optimization", Journal of Structural Engineering, 130(5), pp. 741-751, 2004.

https://doi.org/10.1061/(ASCE)0733-9445(2004)130:5(741)

[42] Camp, C. V. "Design of Space Trusses Using Big Bang-Big Crunch Optimization", Journal of Structural Engineering, 133(7), pp. 9991008, 2007. https://doi.org/10.1061/(ASCE)0733-9445(2007)133:7(999)

[43] Camp, C. V., Farshchin, M. "Design of space trusses using modified teaching-learning based optimization", Engineering Structures, 62-63, pp. 87-97, 2014.

https://doi.org/10.1016/j.engstruct.2014.01.020

[44] Shourestani, S., Soltani, F., Ghasemi, M., Etedali, S. "SSI effects on seismic behavior of smart base-isolated structures", Geomechanics and Engineering, 14(2), pp. 161-174, 2018. https://doi.org/10.12989/gae.2018.14.2.161

[45] Lee, C.-L., Chen, Y.-T., Chung, L.-L., Wang, Y.-P. "Optimal design theories and applications of tuned mass dampers", Engineering Structures, 28(1), pp. 43-53, 2006. https://doi.org/10.1016/j.engstruct.2005.06.023

[46] Asai, T. "Structural control strategies for earthquake response reduction of buildings", PhD Dissertation, University of Illinois at Urbana-Champaign, 2014. [online] Available at: https://www.ideals.illinois.edu/handle/2142/49571 